ESAIM: PROCEEDINGS, March 2012, Vol. 35, p. 14-45

Fédération Denis Poisson (Orléans-Tours) et E. Trélat (UPMC), Editors

\title{
QUANTUM WAVEGUIDES WITH CORNERS
}

\author{
Monique Dauge ${ }^{1}$, Yvon Lafranche ${ }^{1}$ and Nicolas Raymond ${ }^{1}$
}

\begin{abstract}
The simplest modeling of planar quantum waveguides is the Dirichlet eigenproblem for the Laplace operator in unbounded open sets which are uniformly thin in one direction. Here we consider V-shaped guides. Their spectral properties depend essentially on a sole parameter, the opening of the $\mathrm{V}$. The free energy band is a semi-infinite interval bounded from below. As soon as the $\mathrm{V}$ is not flat, there are bound states below the free energy band. There are a finite number of them, depending on the opening. This number tends to infinity as the opening tends to 0 (sharply bent V). In this situation, the eigenfunctions concentrate and become self-similar. In contrast, when the opening gets large (almost flat V), the eigenfunctions spread and enjoy a different self-similar structure. We explain all these facts and illustrate them by numerical simulations.
\end{abstract}

Résumé. La modélisation la plus simple des guides d'ondes quantiques plans est le problème aux valeurs propres pour le laplacien dans des ouverts non bornés qui sont fins dans une direction. Ici nous considérons des guides en forme de V. Leurs propriétés spectrales dépendent essentiellement d'un seul paramètre, l'ouverture du V. La bande d'énergie libre est un intervalle semi-infini borné inférieurement. Dès que le V n'est pas plat, il existe des états liés sous la bande d'énergie libre. Ils sont en nombre fini, fonction de l'ouverture. Ce nombre tend vers l'infini quand l'ouverture tend vers 0 ( $\mathrm{V}$ très refermé). Dans cette situation, les fonctions propres se concentrent et deviennent autosimilaires. À l'opposé, quand l'ouverture est grande (V très aplati), les fonctions propres s'étalent et jouissent d'une autre structure auto-similaire. Nous expliquons tous ces résultats et les illustrons par des expériences numériques.

\section{INTRODUCTION}

A quantum waveguide refer to nanoscale electronic device with a wire or thin surface shape. In the first case, one speaks of a quantum wire. The electronic density is low enough to allow a modeling of the system by a simple one-body Schrödinger operator with potential

$$
\psi \longmapsto-\Delta \psi+V \psi \quad \text { in } \quad \mathbb{R}^{3} .
$$

The structure of the device causes the potential to be very large outside and very small inside the device. As a relevant approximation, we can consider that the potential is zero in the device and infinite outside ; this can be described by a Dirichlet operator

$$
\psi \longmapsto-\Delta \psi \text { in } \Omega \text { and } \psi=0 \text { on } \partial \Omega
$$

\footnotetext{
1 IRMAR (UMR CNRS 6625), Université de Rennes 1, France

e-mail: monique.dauge@univ-rennes1.fr,

e-mail: yvon.lafranche@univ-rennes1.fr,

e-mail: nicolas.raymondQuniv-rennes1.fr
}

(C) EDP Sciences, SMAI 2012 


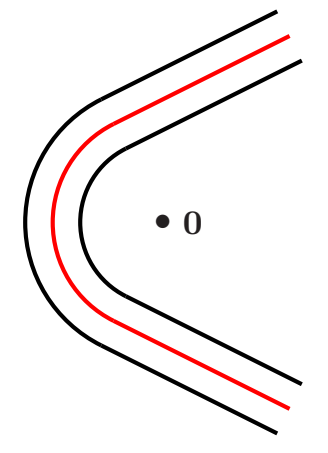

Figure 1. Curved guide

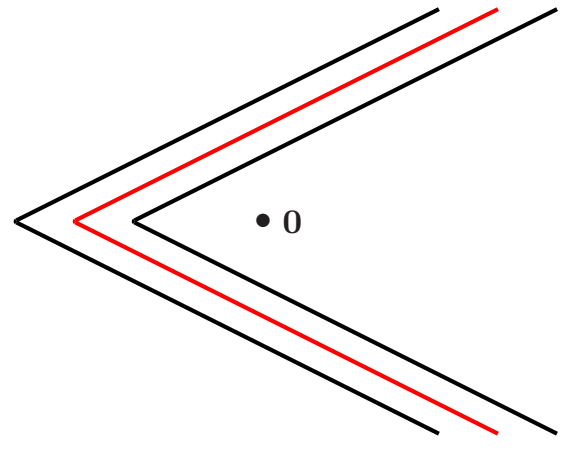

Figure 2. Broken guide

where $\Omega$ is the open set filled by the device. We refer to [3] where we can see, at least on the numerical simulations, the analogy between a problem with a confining potential and a Dirichlet condition.

These kinds of device are intended to drive electronic fluxes. But their shape may capture some bound states, i.e. eigenpairs of the Dirichlet problem:

$$
-\Delta \psi=\lambda \psi \text { in } \Omega \text { and } \psi=0 \text { on } \partial \Omega .
$$

The topic of this paper is two-dimensional wire shaped structures, i.e. structures which coincide with strips of the form $\mathbb{R}_{+} \times(0, \alpha)$ outside a ball of center $\mathbf{0}$ and radius $R$ large enough. These structures can be called planar waveguides. More specifically, there are the bent waveguides and the broken waveguides: Bent waveguides have a constant width around some central smooth curve, see Figure 1, and the central curve of a broken waveguide is a broken line, see Figure 2.

Due to the semi-infinite strips contained in such waveguide, the spectrum of the Laplacian $-\Delta$ with Dirichlet conditions is not discrete: It contains a semi-infinite interval of the form $[\mu,+\infty)$ which is the energy band where electronic transport can occur. The presence of discrete spectrum at lower energy levels is not obvious, but nevertheless, frequent.

A remarkable result by Duclos and Exner [10] (and generalized in [6]) tells us that if the mid-line of a planar waveguide is smooth and straight outside a compact set, then there exists bound states as soon as the line is not straight everywhere. For broken guides, a similar result holds, [2,11]: There exist bound states as soon as the guide is not a straight strip. The question of the increasing number of such bound states for sharply bent broken waveguides has been answered in [5].

Before stating the main results of this paper, let us mention a few other works involving broken waveguides such as $[15,16]$. It also turns out that our analysis leads to the investigation of triangles with a sharp angle as in [14] (see also [4]). The generalization to dimension 3 of the broken strip arises in [12] where a conical waveguide is studied, whereas a Born-Oppenheimer approach is in progress in [28].

In this paper, we revisit the results of $[2,5]$ and prove several other quantitative or qualitative properties of the eigenpairs of planar broken waveguides. Here are the contents of the present work: In section 1 we recall from the literature notions of unbounded self-adjoint operators, discrete and essential spectrum, Rayleigh quotients. After proving that the Rayleigh quotients are increasing functions of the opening angle $\theta$ of the guide (section 3), we adapt the technique of [6] to give a self-contained proof of the existence of bound states (section 4). We prove that the number of bound states is always finite, though depending on the opening angle $\theta$ (section 5), that this number tends to infinity like the inverse $\theta^{-1}$ of the opening angle when $\theta \rightarrow 0$ (section 7). Concerning eigenvectors we prove that they satisfy an even symmetry property with respect to the symmetry axis of the guide (section 2). Their decay along the semi-infinite straight parts of the guide can be precisely evaluated (section 6) and is stronger and stronger when $\theta$ decreases. We perform numerical computations by the finite element method, which clearly illustrate these decay properties. When the opening gets small, concentration 
and self-similarity appears, which can be explained by a semi-classical analysis: We give some overview of the asymptotic expansions established in our other paper [9]. We end this work by evaluations of the numerical convergence of the algorithms used for our finite element computations (section 9).

Notation. The $\mathrm{L}^{2}$ norm on an open set $\mathcal{U}$ will be denoted by $\|\cdot\|_{\mathcal{U}}$.

\section{UNBOUNDED SELF-ADJOINT OPERATORS}

In this section we recall from the literature some definitions and fundamental facts on unbounded self-adjoint operators and their spectrum. We quote the standard book of Reed and Simon [30, Chapter VIII] and, for the readers who can read french, the book of Lévy-Bruhl [23] (see in particular Chapter 10 on unbounded operators).

Let $H$ be a separable Hilbert space with scalar product $\langle\cdot, \cdot\rangle_{H}$. We will consider operators $A$ defined on a dense subspace $\operatorname{Dom}(A)$ of $H$ called the domain of $A$. The adjoint $A^{*}$ of $A$ is the operator defined as follows:

(i) The domain $\operatorname{Dom}\left(A^{*}\right)$ is the space of the elements $u$ of $H$ such that the form

$$
\operatorname{Dom}(A) \ni v \mapsto\langle u, A v\rangle_{H}
$$

can be extended to a continuous form on $H$.

(ii) For any $u \in \operatorname{Dom}\left(A^{*}\right), A^{*} u$ is the unique element of $H$ provided by the Riesz theorem such that

$$
\forall v \in \operatorname{Dom}(A), \quad\left\langle A^{*} u, v\right\rangle_{H}=\langle u, A v\rangle_{H}
$$

Definition 1.1. The operator $A$ with domain $\operatorname{Dom}(A)$ is said self-adjoint if $A=A^{*}$, which means that

$$
\operatorname{Dom}\left(A^{*}\right)=\operatorname{Dom}(A) \quad \text { and } \quad \forall u, v \in \operatorname{Dom}(A), \quad\langle A u, v\rangle_{H}=\langle u, A v\rangle_{H} .
$$

\subsection{Operators in variational form}

The operators that we will consider can be defined by variational formulation. Let us introduce the general framework first. Let be given two separable Hilbert spaces $H$ and $V$ with continuous embedding of $V$ into $H$ and such that $V$ is dense in $H$. Let $b$ be an hermitian sesquilinear form on $V$

$$
b: V \times V \ni(u, v) \mapsto b(u, v) \in \mathbb{C}
$$

which is assumed to be continuous and coercive: This means that there exist three real numbers $c, C$ and $\Lambda$ such that

$$
\forall u \in V, \quad c\|u\|_{V}^{2} \leq b(u, u)+\Lambda\langle u, u\rangle_{H} \leq C\|u\|_{V}^{2} .
$$

Let $\underline{A}$ be the operator defined from $V$ into its dual $V^{\prime}$ by the natural expression

$$
\forall v \in V, \quad\langle\underline{A} u, v\rangle_{H}=b(u, v) .
$$

In other words, for all $u \in V, \underline{A} u$ is the linear form $v \mapsto b(u, v)$. Note that the operator $\underline{A}+\Lambda$ Id is associated in the same way to the sesquilinear form

$$
b+\Lambda\langle\cdot, \cdot\rangle_{H}: V \times V \ni(u, v) \mapsto b(u, v)+\Lambda\langle u, v\rangle_{H} \in \mathbb{C}
$$

which is strongly elliptic by (3). As a consequence of the Riesz theorem (or the more general Lax-Milgram theorem) there holds

$$
\underline{A}+\Lambda \mathrm{Id} \text { is an isomorphism from } V \text { onto } V^{\prime} \text {. }
$$

This situation provides many examples of (unbounded) self-adjoint operators. The following lemma is related to the Friedrichs's lemma $c f$. [23, Section 10.7]. 
Lemma 1.2. Let $\underline{A}$ be the operator associated with an hermitian sesquilinear form $b$ coercive on $V$. Let $A$ be the restriction of the operator $\underline{A}$ on the domain

$$
\operatorname{Dom}(A)=\{u \in V: \quad \underline{A} u \in H\}
$$

Then $A$ is self-adjoint.

Proof. The operator $A$ is symmetric because $b$ is hermitian. Moreover we check immediately that

$$
\forall u, v \in \operatorname{Dom}(A), \quad\langle A u, v\rangle_{H}=\langle u, A v\rangle_{H}=b(u, v) .
$$

In particular, we deduce $\operatorname{Dom}(A) \subset \operatorname{Dom}\left(A^{*}\right)$. Let us prove that $\operatorname{Dom}\left(A^{*}\right) \subset \operatorname{Dom}(A)$. Since the domain of $A$ and $A^{*}$ are unchanged by the addition of $\Lambda \mathrm{Id}$, and since

$$
(A+\Lambda \mathrm{Id})^{*}=A^{*}+\Lambda \mathrm{Id}
$$

we can consider $A+\Lambda$ Id instead of $A$, or, in other words, using (4), assume that $\underline{A}$ is bijective.

Then we deduce that $A$ is an isomorphism from $\operatorname{Dom}(A)$ onto $H$ : Indeed, $A$ is injective because $\underline{A}$ is injective; If $f \in H$, there exists $u \in V$ such that $\underline{A} u=f$, and $u \in \operatorname{Dom}(A)$ by definition of $\operatorname{Dom}(A)$.

Let $w$ belong to $\operatorname{Dom}\left(A^{*}\right)$. This means, $c f$. (2), that $w \in H$ and $A^{*} w=f \in H$. Let us prove that $w$ belongs to $\operatorname{Dom}(A)$.

Since $A$ is bijective there exists $u \in \operatorname{Dom}(A)$ such that $A u=f$. We have

$$
\forall v \in V, \quad b(u, v)=\langle f, v\rangle_{H}
$$

and therefore

$$
\forall v \in \operatorname{Dom}(A), \quad\langle u, A v\rangle_{H}=\langle w, A v\rangle_{H} .
$$

Hence, as $A$ is bijective, for all $g \in H,\langle u, g\rangle_{H}=\langle w, g\rangle_{H}$. Finally $u=w$, which ends the proof.

Example 1.3. Let $\Omega$ be an open set in $\mathbb{R}^{n}$ and $\partial_{\mathrm{Dir}} \Omega$ a part of its boundary. On

$$
V=\left\{\psi \in \mathrm{H}^{1}(\Omega): \quad \psi=0 \text { on } \partial_{\mathrm{Dir}} \Omega\right\}
$$

we consider the bilinear form

$$
b\left(\psi, \psi^{\prime}\right)=\int_{\Omega} \nabla \psi(\mathbf{x}) \cdot \nabla \psi^{\prime}(\mathbf{x}) \mathrm{d} \mathbf{x} .
$$

The operator $A$ is equal to $-\Delta$ and it is self-adjoint on $H=\mathrm{L}^{2}(\Omega)$ with domain

$$
\operatorname{Dom}(A)=\left\{\psi \in V: \quad \Delta \psi \in \mathrm{L}^{2}(\Omega) \quad \text { and } \quad \partial_{n} \psi=0 \quad \text { on } \partial \Omega \backslash \partial_{\mathrm{Dir}} \Omega\right\} .
$$

\subsection{Discrete and essential spectrum}

Let $A$ be an unbounded self-adjoint operator on $H$ with domain $\operatorname{Dom}(A)$. We recall the following characterizations of its spectrum $\sigma(A)$, its essential spectrum $\sigma_{\text {ess }}(A)$ and its discrete spectrum $\sigma_{\text {dis }}(A)$ :

- Spectrum: $\lambda \in \sigma(A)$ if and only if $(A-\lambda \mathrm{Id})$ is not invertible from $\operatorname{Dom}(A)$ onto $H$,

- Essential spectrum: $\lambda \in \sigma_{\text {ess }}(A)$ if and only if $(A-\lambda$ Id $)$ is not Fredholm ${ }^{1}$ from $\operatorname{Dom}(A)$ into $H$ (see [30, Chapter VI] and [23, Chapter 3]),

- Discrete spectrum: $\sigma_{\text {dis }}(A):=\sigma(A) \backslash \sigma_{\text {ess }}(A)$.

We list now several fundamental properties of essential and discrete spectrum.

\footnotetext{
${ }^{1}$ We recall that an operator is said to be Fredholm if its kernel is finite dimensional, its range is closed and with finite codimension.
} 
Lemma 1.4 (Weyl criterion). We have $\lambda \in \sigma_{\text {ess }}(A)$ if and only if there exists a sequence $\left(u_{n}\right) \in \operatorname{Dom}(A)$ such that $\left\|u_{n}\right\|_{H}=1,\left(u_{n}\right)$ has no subsequence converging in $H$ and $(A-\lambda \mathrm{Id}) u_{n} \underset{n \rightarrow+\infty}{\rightarrow} 0$ in $H$.

From this lemma, one can deduce (see [23, Proposition 2.21 and Proposition 3.11]):

Lemma 1.5. The discrete spectrum is formed by isolated eigenvalues of finite multiplicity.

Lemma 1.6. The essential spectrum is stable under any perturbation which is compact from $\operatorname{Dom}(A)$ into $H$.

Example 1.7. Let $A$ be the self-adjoint operator on $H$ associated with an hermitian sesquilinear form $b$ coercive on $V, c f$. Lemma 1.2. Let us assume that $V$ is compactly embedded in $H$. Then the spectrum of $A$ is discrete and formed by a non-decreasing sequence $\nu_{k}$ of eigenvalues which tend to $+\infty$ as $k \rightarrow+\infty$ (see [23, Chapter $13])$. Let $\left(v_{k}\right)_{k \geq 1}$ be an associated orthonormal basis of eigenvectors:

$$
A v_{k}=\nu_{k} v_{k}, \quad \forall k \geq 1 .
$$

Then we have the following identities

$$
\begin{gathered}
\forall u \in H, \quad\|u\|_{H}^{2}=\sum_{k \geq 1}\left|\left\langle u, v_{k}\right\rangle_{H}\right|^{2}, \\
\forall u \in V, \quad b(u, u)=\sum_{k \geq 1} \nu_{k}\left|\left\langle u, v_{k}\right\rangle_{H}\right|^{2}, \\
\forall u \in \operatorname{Dom}(A), \quad\|A u\|_{H}^{2}=\sum_{k \geq 1} \nu_{k}^{2}\left|\left\langle u, v_{k}\right\rangle_{H}\right|^{2} .
\end{gathered}
$$

Example 1.8. Let us define $\Delta_{\Omega}^{\text {Dir }}$ as the Dirichlet problem for the Laplace operator $-\Delta$ on an open set $\Omega \subset \mathbb{R}^{n}$, cf. Example 1.3 with $\partial_{\operatorname{Dir}} \Omega=\partial \Omega$.

(i) If $\Omega$ is bounded, $\Delta_{\Omega}^{\text {Dir }}$ has a purely discrete spectrum, which is an increasing sequence of positive numbers.

(ii) Let us assume that there is a compact set $K$ such that

$$
\Omega \backslash K=\bigcup_{j \text { finite }} \Omega_{j} \quad \text { (disjoint union) }
$$

where $\Omega_{j}$ is isometrically affine to a half-tube $\Sigma_{j}=(0,+\infty) \times \omega_{j}$, with $\omega_{j}$ bounded open set in $\mathbb{R}^{n-1}$. Let $\mu_{j}$ be the first eigenvalue of the Dirichlet problem for the Laplace operator $-\Delta$ on $\omega_{j}$. Then, we have:

$$
\sigma_{\mathrm{ess}}\left(\Delta_{\Omega}^{\text {Dir }}\right)=\cup_{j}\left[\mu_{j},+\infty\right)=\left[\min _{j} \mu_{j},+\infty\right)
$$

The proof can be organized in two main steps. Firstly, for each $j$ we construct Weyl sequences supported in $\Sigma_{j}$ associated with any $\lambda>\mu_{j}$, which proves that $\sigma_{\text {ess }}\left(\Delta_{\Omega}^{\text {Dir }}\right) \subset\left[\min _{j} \mu_{j},+\infty\right)$. Secondly we apply Lemma 1.6 with $A=B-C$ where $A=\Delta_{\Omega}^{\text {Dir }}$ and $B=\Delta_{\Omega}^{\text {Dir }}+W$, where $W$ is a non negative and smooth potential which is compactly supported and such that $W \geq \min _{j} \mu_{j}$ on $K$. On one hand, since $C$ is compact, we get that $\sigma_{\mathrm{ess}}(A)=\sigma_{\mathrm{ess}}(B)$. On the other hand, we notice that:

$$
\int_{\Omega}\|\nabla \psi\|^{2} \mathrm{~d} \mathbf{x}+\int_{\Omega} W|\psi|^{2} \mathrm{~d} \mathbf{x} \geq \int_{\Omega}\|\nabla \psi\|^{2} \mathrm{~d} \mathbf{x}+\min _{j} \mu_{j} \int_{K}|\psi|^{2} \mathrm{~d} \mathbf{x}
$$

and, using the Poincaré inequality with respect to the transversal variable in each strip $\Sigma_{j}$ :

$$
\int_{\Omega}\|\nabla \psi\|^{2} \mathrm{~d} \mathbf{x} \geq \sum_{j} \int_{\Sigma j}\|\nabla \psi\|^{2} \mathrm{~d} \mathbf{x} \geq \sum_{j} \int_{\Sigma j} \mu_{j}|\psi|^{2} \mathrm{~d} \mathbf{x} \geq \min _{j} \mu_{j} \int_{\Omega \backslash K}|\psi|^{2} .
$$


We infer that:

$$
\int_{\Omega}\|\nabla \psi\|^{2} \mathrm{~d} \mathbf{x}+\int_{\Omega} W|\psi|^{2} \mathrm{~d} \mathbf{x} \geq \min _{j} \mu_{j} \int_{\Omega}|\psi|^{2} \mathrm{~d} \mathbf{x} .
$$

The min-max principle provides that $\inf \sigma(B) \geq \min _{j} \mu_{j}$ so that $\inf \sigma_{\text {ess }}(B) \geq \min _{j} \mu_{j}$, and finally we get $\inf \sigma_{\text {ess }}(A) \geq \min _{j} \mu_{j}$.

For an example of this technique, we refer for instance to [6, Section 3.1]. Let us notice that the Persson's theorem provides a direct proof (see [29] and [13, Appendix B]).

The same formula holds even if the boundary conditions on $\partial \Omega \cap K$ are mixed Dirichlet-Neumann.

\subsection{Rayleigh quotients}

We recall now the definition of the Rayleigh quotients of a self-adjoint operator $A$ (see [23, Proposition 6.17 and 13.1]).

Definition 1.9. The Rayleigh quotients associated to the self-adjoint operator $A$ on $H$ of domain $\operatorname{Dom}(A)$ are defined for all positive natural number $j$ by

$$
\lambda_{j}=\inf _{\substack{u_{1}, \ldots, u_{j} \in \operatorname{Dom}(A) \\ \text { independent }}} \sup _{u \in\left[u_{1}, \ldots, u_{j}\right]} \frac{\langle A u, u\rangle_{H}}{\langle u, u\rangle_{H}} .
$$

Here $\left[u_{1}, \ldots, u_{j}\right]$ denotes the subspace generated by the $j$ independent vectors $u_{1}, \ldots, u_{j}$.

The following statement gives the relation between Rayleigh quotients and eigenvalues.

Theorem 1.10. Let $A$ be a self-adjoint operator of domain $\operatorname{Dom}(A)$. We assume that $A$ is semi-bounded from below, i.e., there exists $\Lambda \in \mathbb{R}$ such that

$$
\forall u \in \operatorname{Dom}(A), \quad\langle A u, u\rangle_{H}+\Lambda\langle u, u\rangle_{H} \geq 0 .
$$

We set $\gamma=\min \sigma_{\text {ess }}(A)$. Then the Rayleigh quotients $\lambda_{j}$ of $A$ form a non-decreasing sequence and there holds

(i) If $\lambda_{j}<\gamma$, it is an eigenvalue of $A$,

(ii) If $\lambda_{j} \geq \gamma$, then $\lambda_{j}=\gamma$,

(iii) The $j$-th eigenvalue $<\gamma$ of $A$ (if exists) coincides with $\lambda_{j}$.

Lemma 1.11. Let $A$ be the self-adjoint operator on $H$ associated with an hermitian sesquilinear form $b$ coercive on $V$, cf. Lemma 1.2. Then the Rayleigh quotients of $A$ are equal to

$$
\lambda_{j}=\inf _{\substack{u_{1}, \ldots, u_{j} \in V \\ \text { independent }}} \sup _{\substack{u \in\left[u_{1}, \ldots, u_{j}\right]\\}} \frac{b(u, u)}{\langle u, u\rangle_{H}} .
$$

Corollary 1.12. Let $A$ and $\hat{A}$ be the self-adjoint operators on $H$ and $\hat{H}$ associated with the hermitian sesquilinear forms $b$ and $\hat{b}$ coercive on $V$ and $\hat{V}$, respectively. We assume that

$$
\hat{H} \subset H, \quad \hat{V} \subset V, \quad \hat{b}(u, u) \geq b(u, u) \forall u \in \hat{V} .
$$

Let $\lambda_{j}$ and $\hat{\lambda}_{j}$ be the Rayleigh quotients associated to $A$ and $\hat{A}$, respectively. Then

$$
\forall j \geq 1, \quad \hat{\lambda}_{j} \geq \lambda_{j}
$$

Example 1.13 (Conforming Galerkin projection). This consists in choosing a finite dimensional subspace $\hat{V}$ of $V$, which also defines a subspace of $H$, and $\hat{b}=b$. The Rayleigh quotients $\hat{\lambda}_{j}$ are the eigenvalues of the discrete operator $\hat{A}$, and they are larger than the Rayleigh quotients $\lambda_{j}$ of $A$. 
Example 1.14 (Monotonicity of Dirichlet eigenvalues). Let $\Omega$ be an open subset of $\mathbb{R}^{n}$ and $\widehat{\Omega} \subset \Omega$. The extension by 0 from $\widehat{\Omega}$ to $\Omega$ realizes a natural embedding of $\mathrm{H}_{0}^{1}(\widehat{\Omega})$ into $\mathrm{H}_{0}^{1}(\Omega)$, and of $\mathrm{L}^{2}(\widehat{\Omega})$ into $\mathrm{L}^{2}(\Omega)$. Then, with obvious notations:

$$
\forall j \geq 1, \quad \lambda_{j}\left(\Delta_{\widehat{\Omega}}^{\text {Dir }}\right) \geq \lambda_{j}\left(\Delta_{\Omega}^{\text {Dir }}\right)
$$

Remark 1.15 (Non-monotonicity of Neumann eigenvalues). The Neumann problem consists in taking $H^{1}(\Omega)$ as variational space. The argument above does not work because there is no canonical embedding of $H^{1}(\widehat{\Omega})$ into $H^{1}(\Omega)$. Moreover, the monotonicity with respect to the domain is wrong:

(i) Let us choose $\Omega$ bounded and connected, and $\widehat{\Omega}$ a subset of $\Omega$ with two connected components. Then

$$
\lambda_{1}\left(\Delta_{\widehat{\Omega}}^{\text {Neu }}\right)=\lambda_{2}\left(\Delta_{\widehat{\Omega}}^{\text {Neu }}\right)=0 \quad \text { and } \quad \lambda_{1}\left(\Delta_{\Omega}^{\text {Neu }}\right)=0, \lambda_{2}\left(\Delta_{\Omega}^{\text {Neu }}\right)>0 .
$$

(ii) If we take two embedded intervals for $\widehat{\Omega}$ and $\Omega$, then explicit calculations show that $\lambda_{j}\left(\Delta_{\widehat{\Omega}}^{\text {Neu }}\right) \geq \lambda_{j}\left(\Delta_{\Omega}^{\text {Neu }}\right)$.

Another nice and non trivial counter-example can be found with the de Gennes operator appearing in the superconductivity theory, $c f$. [8].

\section{THE BROKEN GUIDE}

Let us denote the Cartesian coordinates in $\mathbb{R}^{2}$ by $\mathbf{x}=\left(x_{1}, x_{2}\right)$. The open sets $\Omega$ that we consider are unbounded plane $\mathrm{V}$-shaped sets. The question of interest is the presence and the properties of bound states for the Laplace operator $\Delta=\partial_{1}^{2}+\partial_{2}^{2}$ with Dirichlet boundary conditions in such $\Omega$. We can assume without loss of generality that our set $\Omega$ is normalized so that

- it has its non-convex corner at the origin $\mathbf{0}=(0,0)$,

- it is symmetric with respect to the $x_{1}$ axis,

- its thickness is equal to $\pi$.

The sole remaining parameter is the opening of the $\mathrm{V}$ : We denote by $\theta \in\left(0, \frac{\pi}{2}\right)$ the half-opening and by $\Omega_{\theta}$ the associated broken guide, see Figure 3. We have

$$
\Omega_{\theta}=\left\{\left(x_{1}, x_{2}\right) \in \mathbb{R}^{2}: \quad x_{1} \tan \theta<\left|x_{2}\right|<\left(x_{1}+\frac{\pi}{\sin \theta}\right) \tan \theta\right\} .
$$

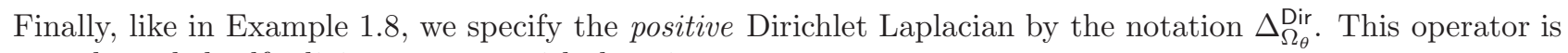
an unbounded self-adjoint operator with domain

$$
\operatorname{Dom}\left(\Delta_{\Omega_{\theta}}^{\text {Dir }}\right)=\left\{\psi \in \mathrm{H}_{0}^{1}\left(\Omega_{\theta}\right): \quad \Delta \psi \in \mathrm{L}^{2}\left(\Omega_{\theta}\right)\right\} .
$$

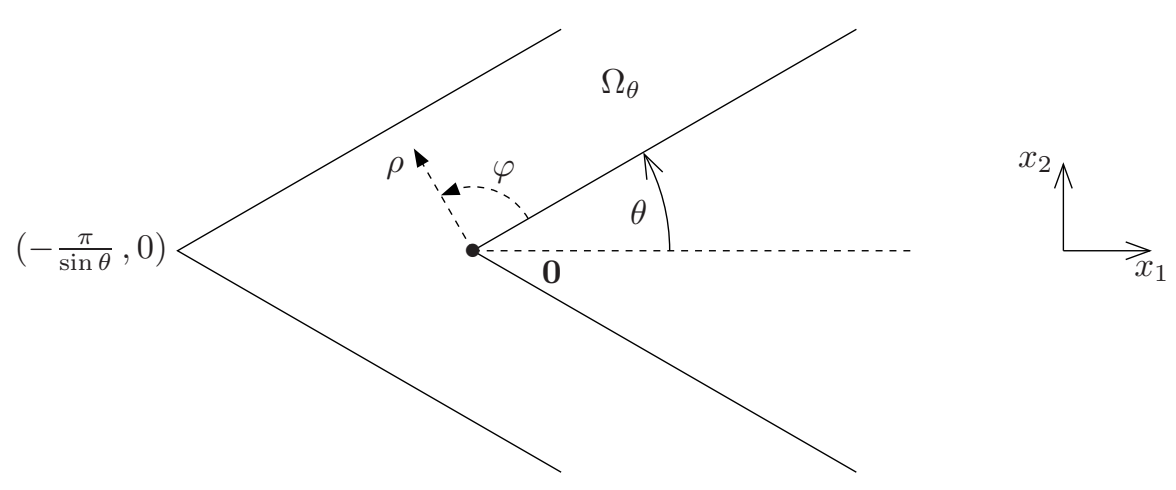

Figure 3. The broken guide $\Omega_{\theta}$ (here $\theta=\frac{\pi}{6}$ ). 
The boundary of $\Omega_{\theta}$ is not smooth, it is polygonal. The presence of the non-convex corner with vertex at the origin is the reason for the domain $\operatorname{Dom}\left(\Delta_{\Omega_{\theta}}^{\text {Dir }}\right)$ to be distinct from $\mathrm{H}^{2} \cap \mathrm{H}_{0}^{1}\left(\Omega_{\theta}\right)$. Nevertheless this domain can be precisely characterized as follows. Let us introduce polar coordinates $(\rho, \varphi)$ centered at the origin, with $\varphi=0$ coinciding with the upper part $x_{2}=x_{1} \tan \theta$ of the boundary of $\Omega_{\theta}$. Let $\chi$ be a smooth radial cutoff function with support in the region $x_{1} \tan \theta<\left|x_{2}\right|$ and $\chi \equiv 1$ in a neighborhood of the origin. We introduce the explicit singular function

$$
\psi_{\text {sing }}\left(x_{1}, x_{2}\right)=\chi(\rho) \rho^{\pi / \omega} \sin \frac{\pi \varphi}{\omega}, \quad \text { with } \quad \omega=2(\pi-\theta) .
$$

Then there holds, $c f$. the classical references [18,22]:

$$
\operatorname{Dom}\left(\Delta_{\Omega_{\theta}}^{\mathrm{Dir}}\right)=\mathrm{H}^{2} \cap \mathrm{H}_{0}^{1}\left(\Omega_{\theta}\right) \oplus\left[\psi_{\text {sing }}\right]
$$

where $\left[\psi_{\text {sing }}\right]$ denotes the space generated by $\psi_{\text {sing }}$.

\subsection{Essential spectrum}

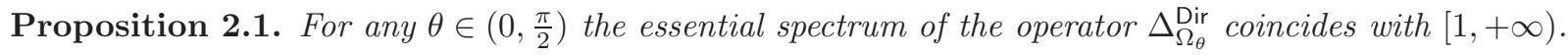

Proof. This proposition is a consequence of Example 1.8 (ii): Outside a compact set, $\Omega_{\theta}$ is the union of two strips isometric to $(0,+\infty) \times(0, \pi)$. Since the first eigenvalue of $-\partial_{y}^{2}$ on $\mathrm{H}_{0}^{1}(0, \pi)$ is 1 , the proposition is proved.

\subsection{Symmetry}

In our quest of bounded states $(\lambda, \psi)$ of $\Delta_{\Omega_{\theta}}^{\text {Dir }}$, i.e.

$$
\left\{\begin{aligned}
-\Delta \psi=\lambda \psi & \text { in } \quad \Omega_{\theta}, \\
\psi=0 & \text { on } \quad \partial \Omega_{\theta}
\end{aligned} \quad \text { with } \quad \lambda<1, \quad \psi \neq 0,\right.
$$

we can reduce to the half-guide $\Omega_{\theta}^{+}$defined as (see Figure 4)

$$
\Omega_{\theta}^{+}=\left\{\left(x_{1}, x_{2}\right) \in \Omega_{\theta}: x_{2}>0\right\}, \quad \text { with the Dirichlet part of its boundary } \partial_{\text {Dir }} \Omega_{\theta}^{+}=\partial \Omega_{\theta} \cap \partial \Omega_{\theta}^{+},
$$

as we are going to explain now. Let us introduce $\Delta_{\Omega_{\theta}^{+}}^{\text {Mix }}$ as the positive Laplacian with mixed Dirichlet-Neumann conditions on $\Omega_{\theta}^{+}$with domain, $c f$. Example 1.3:

$$
\operatorname{Dom}\left(\Delta_{\Omega_{\theta}^{+}}^{\mathrm{Mix}}\right)=\left\{\psi \in H^{1}\left(\Omega_{\theta}^{+}\right): \quad \Delta \psi \in L^{2}\left(\Omega_{\theta}^{+}\right), \quad \psi=0 \quad \text { on } \partial_{\operatorname{Dir}} \Omega_{\theta}^{+} \quad \text { and } \partial_{2} \psi=0 \text { on } x_{2}=0\right\} .
$$

Then $\sigma_{\text {ess }}\left(\Delta_{\Omega_{\theta}^{+}}^{\text {Mix }}\right)$ coincides with $\sigma_{\text {ess }}\left(\Delta_{\Omega_{\theta}}^{\text {Dir }}\right)$. Concerning the discrete spectrum we have:

Proposition 2.2. For any $\theta \in\left(0, \frac{\pi}{2}\right), \sigma_{\mathrm{dis}}\left(\Delta_{\Omega_{\theta}}^{\text {Dir }}\right)$ coincides with $\sigma_{\mathrm{dis}}\left(\Delta_{\Omega_{\theta}^{+}}^{\mathrm{Mix}}\right)$.

Proof. The proof relies on the fact that $\Delta_{\Omega_{\theta}}^{\text {Dir }}$ commutes with the symmetry $S:\left(x_{1}, x_{2}\right) \mapsto\left(x_{1},-x_{2}\right)$.

(i) If $(\lambda, \psi)$ is an eigenpair of $\Delta_{\Omega_{\theta}^{+}}^{\text {Mix }}$, the even extension of $u_{\lambda}$ to $\Omega_{\theta}$ defines an eigenfunction of $\Delta_{\Omega_{\theta}}^{\text {Dir }}$ associated with the same eigenvalue $\lambda$. Therefore we have the inclusion

$$
\sigma_{\mathrm{dis}}\left(\Delta_{\Omega_{\theta}^{+}}^{\mathrm{Mix}}\right) \subset \sigma_{\mathrm{dis}}\left(\Delta_{\Omega_{\theta}}^{\mathrm{Dir}}\right) .
$$

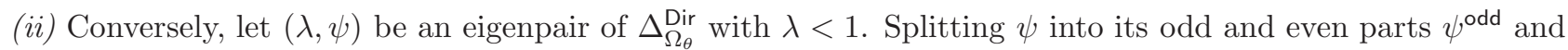
$\psi^{\text {even }}$ with respect to $x_{2}$, we obtain:

$$
\psi=\psi^{\text {odd }}+\psi^{\text {even }}, \quad \Delta_{\Omega_{\theta}}^{\text {Dir }} \psi^{\text {odd }}=\lambda \psi^{\text {odd }} \quad \text { and } \quad \Delta_{\Omega_{\theta}}^{\text {Dir }} \psi^{\text {even }}=\lambda \psi^{\text {even }} .
$$




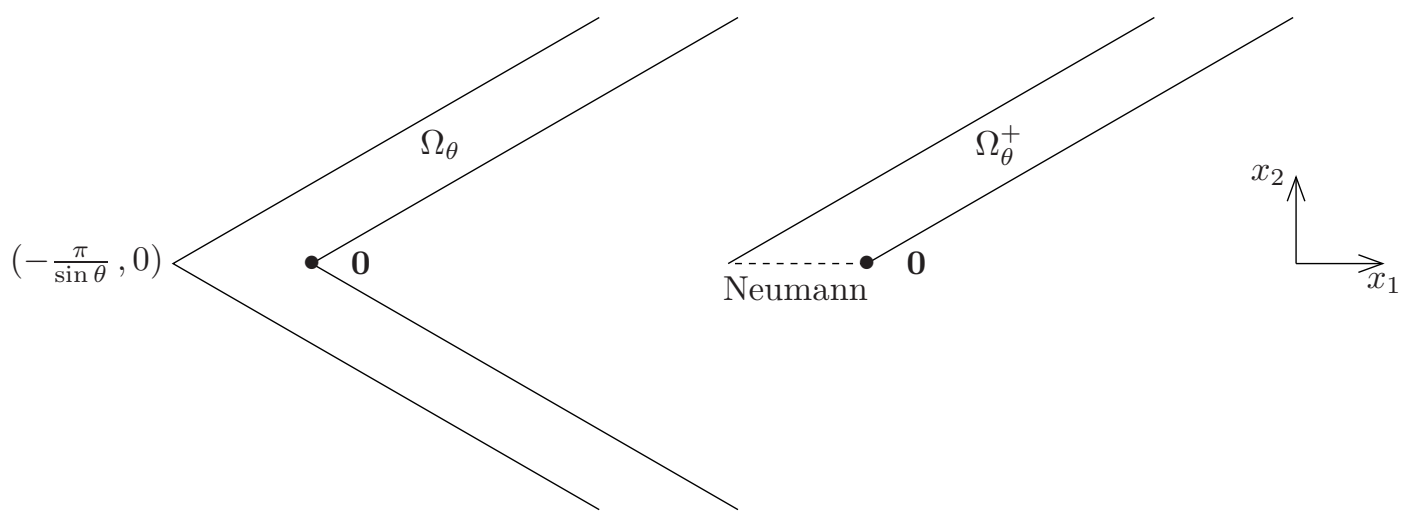

Figure 4 . The waveguide $\Omega_{\theta}$ and the half-guide $\Omega_{\theta}^{+}$(here $\theta=\frac{\pi}{6}$ ).

We note that $\psi^{\text {odd }}$ satisfies the Dirichlet condition on the line $x_{2}=0$, and $\psi^{\text {even }}$ the Neumann condition on the same line. Let us check that $\psi^{\text {odd }}=0$. If it is not the case, this would mean that $\lambda$ is an eigenvalue for the Dirichlet Laplacian on the half-waveguide $\Omega_{\theta}^{+}$. But, by monotonicity of the Dirichlet spectrum with the respect to the domain, $c f$. Example 1.14, we obtain that the spectrum on $\Omega_{\theta}^{+}$is higher than the spectrum on the infinite strip which coincides with $\Omega_{\theta}^{+}$when $x_{1}>0$. This latter spectrum is equal to $[1,+\infty)$. Therefore the Dirichlet Laplacian on the half-waveguide $\Omega_{\theta}^{+}$cannot have an eigenvalue below 1 . Thus, we have necessarily: $\psi^{\text {odd }}=0$ and $\psi=\psi^{\text {even }}$ which is an eigenfunction of $\Delta_{\Omega_{\theta}^{+}}^{\text {Mix }}$ associated with $\lambda$.

We take advantage of Proposition 2.2 for further proofs and for numerical simulations.

\section{Monotonicity of Rayleigh quotients With Respect to the opening}

From now on we consider the Rayleigh quotients associated with the operator $\Delta_{\Omega_{\theta}^{+}}^{\mathrm{Mix}}$, which we write in the form, $c f$. Lemma 1.11

$$
\lambda_{j}(\theta)=\inf _{\substack{\psi_{1}, \ldots, \psi_{j} \text { independent in } \mathrm{H}^{1}\left(\Omega_{\theta}^{+}\right), \psi_{1}, \ldots, \psi_{j}=0 \text { on } \partial_{\operatorname{Dir} \Omega_{\theta}^{+}}}} \sup _{\psi \in\left[\psi_{1}, \ldots, \psi_{j}\right]} \frac{\|\nabla \psi\|_{\Omega_{\theta}^{+}}^{2}}{\|\psi\|_{\Omega_{\theta}^{+}}^{2}}
$$

Those $\lambda_{j}(\theta)$ which are $<1$ are all the eigenvalues of $\Delta_{\Omega_{\theta}}^{\text {Dir }}$ sitting below its essential spectrum.

Proposition 3.1. For any integer $j \geq 1$, the function $\theta \mapsto \lambda_{j}(\theta)$ defined in (12) is non-decreasing from $\left(0, \frac{\pi}{2}\right)$ into $\mathbb{R}_{+}$.

Proof. We cannot use directly Corollary 1.12 because of the part of the boundary where Neumann conditions are prescribed. Instead we introduce the open set $\widetilde{\Omega}_{\theta}$ isometric to $\Omega_{\theta}^{+}$, see Figure 5 ,

$$
\widetilde{\Omega}_{\theta}=\left\{(\tilde{x}, \tilde{y}) \in\left(-\frac{\pi}{\tan \theta},+\infty\right) \times(0, \pi): \quad \tilde{y}<\tilde{x} \tan \theta+\pi \text { if } \tilde{x} \in\left(-\frac{\pi}{\tan \theta}, 0\right)\right\} .
$$

The part $\partial_{\text {Dir }} \widetilde{\Omega}_{\theta}$ of the boundary carrying the Dirichlet condition is the union of its horizontal parts. The numbers $\lambda_{j}(\theta)$ can be equivalently defined by the Rayleigh quotients (12) on $\widetilde{\Omega}_{\theta}$.

Let us now perform the change of variable:

$$
x=\tilde{x} \tan \theta, \quad y=\tilde{y},
$$




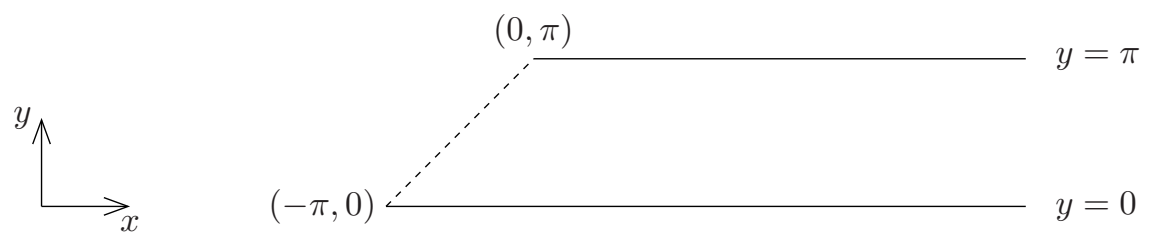

FiguRE 5. The reference half-guide $\widetilde{\Omega}:=\widetilde{\Omega}_{\pi / 4}$.

so that the new integration domain $\widetilde{\Omega}:=\widetilde{\Omega}_{\pi / 4}$ is independent of $\theta$. The bilinear gradient form $b$ on $\widetilde{\Omega}_{\theta}$ is transformed into the anisotropic form $b_{\theta}$ on the fixed set $\widetilde{\Omega}$ :

$$
b_{\theta}\left(\psi, \psi^{\prime}\right)=\int_{\widetilde{\Omega}} \tan ^{2} \theta\left(\partial_{x} \psi \partial_{x} \psi^{\prime}\right)+\left(\partial_{y} \psi \partial_{y} \psi^{\prime}\right) \mathrm{d} x \mathrm{~d} y,
$$

with associated form domain

$$
V:=\left\{\psi \in \mathrm{H}^{1}(\widetilde{\Omega}): \psi=0 \text { on } \partial_{\mathrm{Dir}} \widetilde{\Omega}\right\}
$$

independent of $\theta$.

The function $\theta \mapsto \tan ^{2} \theta$ being increasing on $\left(0, \frac{\pi}{2}\right)$, we have

$$
\forall \psi \in V, \quad \theta \mapsto b_{\theta}(\psi, \psi) \text { non-decreasing on }\left(0, \frac{\pi}{2}\right) .
$$

We conclude thanks to Corollary 1.12 .

Remark 3.2. Using the perturbation theory, we also know that, for all $j \geq 1$, the function $\theta \mapsto \lambda_{j}(\theta)$ is continuous with respect to $\theta \in\left(0, \frac{\pi}{2}\right)$. Moreover, $\lambda_{1}$ being simple (as the first eigenvalue of a Laplace-Dirichlet problem), it is analytic because we are in the situation of an analytic family of type $(B)$ (see $[21$, p. 387 and 395]). In fact the numerical simulations lead to think that all the eigenvalues below 1 are simple and thus analytic.

\section{EXISTENCE OF DISCRETE SPECTRUM}

We recall that the lower bound of the essential spectrum of the operator $\Delta_{\Omega_{\theta}^{+}}^{\text {Mix }}$ is 1. Its first Rayleigh quotient is given by, $c f .(12)$,

$$
\lambda_{1}(\theta)=\inf _{\psi \in \mathrm{H}^{1}\left(\Omega_{\theta}^{+}\right), \psi=0 \text { on } \partial_{\mathrm{Dir}} \Omega_{\theta}^{+}} \frac{\|\nabla \psi\|_{\Omega_{\theta}^{+}}^{2}}{\|\psi\|_{\Omega_{\theta}^{+}}^{2}} .
$$

In this section, we prove the following proposition:

Proposition 4.1. For any $\theta \in\left(0, \frac{\pi}{2}\right)$, the first Rayleigh quotient $\lambda_{1}(\theta)$ is $<1$.

This statement implies that $\lambda_{1}(\theta)$ is an eigenvalue of $\Delta_{\Omega_{\theta}^{+}}^{\text {Mix }}$ (application of Theorem 1.10), hence of the Dirichlet Laplacian $\Delta_{\Omega_{\theta}}^{\text {Dir }}$ on the broken guide (Proposition 2.2).

This statement was first established in [2]. Here we present a distinct, more synthetic proof, using the method of $[6$, p. 104-105].

Proof. For convenience, it is easier to work in the reference set $\widetilde{\Omega}=\widetilde{\Omega}_{\pi / 4}$ introduced in the previous section, with the bilinear form $b_{\theta}(13)$ and the form domain $V(14)$. We are going to work with the shifted bilinear form

$$
\tilde{b}_{\theta}\left(\psi, \psi^{\prime}\right)=b_{\theta}\left(\psi, \psi^{\prime}\right)-\int_{\widetilde{\Omega}} \psi \psi^{\prime} \mathrm{d} x \mathrm{~d} y
$$


which is associated with the quadratic form:

$$
\widetilde{Q}_{\theta}(\psi)=\tilde{b}_{\theta}(\psi, \psi)
$$

Then the first Rayleigh quotient of $\widetilde{Q}_{\theta}$ is equal to $\lambda_{1}(\theta)-1$. To prove our statement, this is enough to construct a function $\psi \in V$ such that:

$$
\widetilde{Q}_{\theta}(\psi)<0 .
$$

This will be done by the construction of

(1) A sequence $\psi_{n} \in V$ such that $\widetilde{Q}_{\theta}\left(\psi_{n}\right) \rightarrow 0$ as $n \rightarrow \infty$,

(2) An element $\phi$ of $V$ such that $\tilde{b}_{\theta}\left(\psi_{n}, \phi\right)$ is nonzero and independent of $n$.

The desired function will then be obtained as a suitable combination $\psi_{n}+\varepsilon \phi$. Let us give details now.

SteP 1. In order to do that, we consider the Weyl sequence defined as follows. Let $\chi$ be a smooth cutoff function equal to 1 for $x \leq 0$ and 0 for $x \geq 1$. We let, for $n \in \mathbb{N} \backslash\{0\}$ :

$$
\chi_{n}(x)=\chi\left(\frac{x}{n}\right) \quad \text { and } \quad \psi_{n}(x, y)=\chi_{n}(x) \sin y .
$$

Using the support of $\chi_{n}$, we find that $\widetilde{Q}_{\theta}\left(\psi_{n}\right)$ is equal to

$$
\int_{-\pi}^{0} \int_{0}^{x+\pi}\left(\cos ^{2} y-\sin ^{2} y\right) \mathrm{d} y \mathrm{~d} x+\int_{0}^{\infty} \int_{0}^{\pi}\left(\tan ^{2} \theta\left(\chi_{n}^{\prime}\right)^{2}(x) \sin ^{2} y+\chi_{n}^{2}(x)\left(\cos ^{2} y-\sin ^{2} y\right)\right) \mathrm{d} y \mathrm{~d} x .
$$

Then, elementary computations provide:

$$
\int_{0}^{\pi}\left(\cos ^{2} y-\sin ^{2} y\right) \mathrm{d} y=0 \text { and } \int_{-\pi}^{0} \int_{0}^{x+\pi}\left(\cos ^{2} y-\sin ^{2} y\right) \mathrm{d} y \mathrm{~d} x=0 .
$$

Moreover, we have:

$$
\int_{0}^{\infty} \int_{0}^{\pi} \tan ^{2} \theta\left(\chi_{n}^{\prime}\right)^{2} \sin ^{2} y \mathrm{~d} y \mathrm{~d} x \leq\left(\int_{0}^{1}\left|\chi^{\prime}(u)\right|^{2} \mathrm{~d} u\right) \frac{\pi \tan ^{2} \theta}{2 n} .
$$

Hence we have proved that $\widetilde{Q}_{\theta}\left(\psi_{n}\right)$ tends to 0 as $n \rightarrow \infty$ :

$$
\widetilde{Q}_{\theta}\left(\psi_{n}\right) \leq \frac{K_{\theta}}{2 n} \quad \text { with } \quad K_{\theta}=\left(\int_{0}^{1}\left|\chi^{\prime}(u)\right|^{2} \mathrm{~d} u\right) \pi \tan ^{2} \theta
$$

STEP 2. We introduce a smooth cutoff function $\eta$ of $x$ supported in $(-\pi, 0)$. We consider a function $f$ of $y \in[0, \pi]$ to be determined later and satisfying $f(0)=0$. We define $\phi(x, y)=\eta(x) f(y)$. For $\varepsilon>0$ to be chosen small enough, we introduce:

We have:

$$
\psi_{n, \varepsilon}(x, y)=\psi_{n}(x, y)+\varepsilon \phi(x, y) .
$$

$$
\widetilde{Q}_{\theta}\left(\psi_{n, \varepsilon}\right)=\widetilde{Q}_{\theta}\left(\psi_{n}\right)+2 \varepsilon \tilde{b}_{\theta}\left(\psi_{n}, \phi\right)+\varepsilon^{2} \widetilde{Q}_{\theta}(\phi) .
$$

Let us compute $\tilde{b}_{\theta}\left(\psi_{n}, \phi\right)$. We can write, thanks to considerations of support:

$$
\tilde{b}_{\theta}\left(\psi_{n}, \phi\right)=\int_{-\pi}^{0} \int_{0}^{x+\pi} \eta(x)\left(\cos y f^{\prime}(y)-\sin y f(y)\right) \mathrm{d} y \mathrm{~d} x=\int_{-\pi}^{0} \int_{0}^{x+\pi} \eta(x)(\cos y f(y))^{\prime} \mathrm{d} y \mathrm{~d} x .
$$

Using $f(0)=0$, this leads to:

$$
\tilde{b}_{\theta}\left(\psi_{n}, \phi\right)=\int_{-\pi}^{0} \eta(x) \cos (x+\pi) f(x+\pi) \mathrm{d} x .
$$


We choose $f(y)=\eta(y-\pi) \cos (y-\pi)$ and we find:

$$
\tilde{b}_{\theta}\left(\psi_{n}, \phi\right)=-\int_{-\pi}^{0} \eta^{2}(x) \cos ^{2}(x) \mathrm{d} x=-\Gamma<0 .
$$

This implies, using (16):

$$
\widetilde{Q}_{\theta}\left(\psi_{n, \varepsilon}\right) \leq \frac{K_{\theta}}{2 n}-2 \Gamma \varepsilon+D \varepsilon^{2},
$$

where $D=\widetilde{Q}_{\theta}(\phi)$ is independent of $\varepsilon$ and $n$. There exists $\varepsilon>0$ such that:

$$
-2 \Gamma \varepsilon+D \varepsilon^{2} \leq-\Gamma \varepsilon .
$$

The angle $\theta$ being fixed, we can take $N$ large enough so that

$$
\frac{K_{\theta}}{2 N} \leq \frac{\Gamma}{2} \varepsilon
$$

from which we deduce that $\widetilde{Q}_{\theta}\left(\psi_{N, \varepsilon}\right) \leq-\varepsilon \Gamma / 2<0$, which ends the proof of Proposition 4.1.

\section{Finite number of eigenvalues Below the ESSEntial SPeCtrum}

For a self-adjoint operator $A$ and a chosen real number $\lambda$ we denote by $\mathcal{N}(A, \lambda)$ the maximal index $j$ such that the $j$-th Rayleigh quotient of $A$ is $<\lambda$. By extension of notation, if the operator $A$ is defined by a coercive hermitian form $b$ on a form domain $V$, and if $Q$ denotes the associated quadratic form $Q(u)=b(u, u)$, we also denote by $\mathcal{N}(Q, \lambda)$ the number $\mathcal{N}(A, \lambda)$. This is coherent with the fact that in this case the Rayleigh quotients can be defined directly by $Q, c f$. Lemma 1.11 :

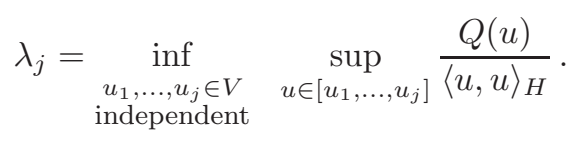

This section is devoted to the proof of the following proposition:

Proposition 5.1. For any $\theta \in\left(0, \frac{\pi}{2}\right), \mathcal{N}\left(\Delta_{\Omega_{\theta}}^{\text {Dir }}, 1\right)$ is finite.

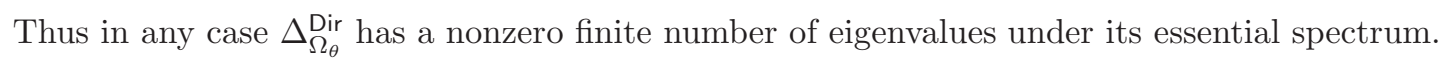

Proof. For the proof of Proposition 5.1 we use a similar method as [27, Theorem 2.1].

Like for the proof of Proposition 4.1, it is easier to work in the reference set $\widetilde{\Omega}$ introduced in section 3 , with the bilinear form $b_{\theta}(13)$ and the form domain $V(14)$. The opening $\theta$ being fixed, we drop the index $\theta$ in the notation of quadratic forms and write simply as $Q$ the quadratic form associated with $b_{\theta}$ :

$$
Q(\psi)=b_{\theta}(\psi, \psi)=\int_{\widetilde{\Omega}} \tan ^{2} \theta\left|\partial_{x} \phi\right|^{2}+\left|\partial_{y} \phi\right|^{2} \mathrm{~d} x \mathrm{~d} y
$$

We recall that the form domain $V$ is the subspace of $\psi \in H^{1}(\widetilde{\Omega})$ which satisfy the Dirichlet condition on $\partial_{\text {Dir }} \widetilde{\Omega}$. We want to prove that

$$
\mathcal{N}(Q, 1) \text { is finite. }
$$

We consider a $\mathrm{C}^{1}$ partition of unity $\left(\chi_{0}, \chi_{1}\right)$ such that

$$
\chi_{0}(x)^{2}+\chi_{1}(x)^{2}=1
$$


with $\chi_{0}(x)=1$ for $x<1$ and $\chi_{0}(x)=0$ for $x>2$. For $R>0$ and $\ell \in\{0,1\}$, we introduce:

$$
\chi_{\ell, R}(x)=\chi_{\ell}\left(R^{-1} x\right) .
$$

Thanks to the IMS formula (see for instance [7]), we can split the quadratic form as:

$$
Q(\psi)=Q\left(\chi_{0, R} \psi\right)+Q\left(\chi_{1, R} \psi\right)-\left\|\chi_{0, R}^{\prime} \psi\right\|_{\widetilde{\Omega}}^{2}-\left\|\chi_{1, R}^{\prime} \psi\right\|_{\widetilde{\Omega}}^{2} .
$$

We can write

$$
\left|\chi_{0, R}^{\prime}(x)\right|^{2}+\left|\chi_{1, R}^{\prime}(x)\right|^{2}=R^{-2} W_{R}(x) \quad \text { with } \quad W_{R}(x)=\left|\chi_{0}^{\prime}\left(R^{-1} x\right)\right|^{2}+\left|\chi_{1}^{\prime}\left(R^{-1} x\right)\right|^{2} .
$$

Then

$$
\begin{aligned}
\left\|\chi_{0, R}^{\prime} \psi\right\|_{\widetilde{\Omega}}^{2}+\left\|\chi_{1, R}^{\prime} \psi\right\|_{\widetilde{\Omega}}^{2} & =\int_{\widetilde{\Omega}} R^{-2} W_{R}(x)|\psi|^{2} \mathrm{~d} x \mathrm{~d} y \\
& =\int_{\widetilde{\Omega}} R^{-2} W_{R}(x)\left(\left|\chi_{0, R} \psi\right|^{2}+\left|\chi_{1, R} \psi\right|^{2}\right) \mathrm{d} x \mathrm{~d} y .
\end{aligned}
$$

Let us introduce the subsets of $\widetilde{\Omega}$ :

$$
\mathcal{O}_{0, R}=\{(x, y) \in \widetilde{\Omega}: x<2 R\} \quad \text { and } \quad \mathcal{O}_{1, R}=\{(x, y) \in \widetilde{\Omega}: x>R\}
$$

and the associated form domains

$$
\begin{gathered}
V_{0}=\left\{\phi \in H^{1}\left(\mathcal{O}_{0, R}\right): \quad \phi=0 \text { on } \partial_{\operatorname{Dir}} \widetilde{\Omega} \cap \partial \mathcal{O}_{0, R} \text { and on }\{2 R\} \times(0, \pi)\right\} \\
V_{1}=\mathrm{H}_{0}^{1}\left(\mathcal{O}_{1, R}\right) .
\end{gathered}
$$

We define the two quadratic forms $Q_{0, R}$ and $Q_{1, R}$ by

$$
Q_{\ell, R}(\phi)=\int_{\mathcal{O}_{\ell, R}} \tan ^{2} \theta\left|\partial_{x} \phi\right|^{2}+\left|\partial_{y} \phi\right|^{2}-R^{-2} W_{R}(x)|\phi|^{2} \mathrm{~d} x \mathrm{~d} y \quad \text { for } \quad \psi \in V_{\ell}, \quad \ell=0,1 .
$$

As a consequence of (17) and (18) we find

$$
Q(\psi)=Q_{0, R}\left(\chi_{0, R} \psi\right)+Q_{1, R}\left(\chi_{1, R} \psi\right) \quad \forall \psi \in V .
$$

Let us prove

Lemma 5.2. We have:

$$
\mathcal{N}(Q, 1) \leq \mathcal{N}\left(Q_{0, R}, 1\right)+\mathcal{N}\left(Q_{1, R}, 1\right) .
$$

Proof. We recall the formula for the $j$-th Rayleigh quotient of $Q$ :

$$
\lambda_{j}=\inf _{\substack{E \subset V \\ \operatorname{dim} E=j}} \sup _{\psi \in E} \frac{Q(\psi)}{\|\psi\|_{\widetilde{\Omega}}^{2}} .
$$

The idea is now to give a lower bound for $\lambda_{j}$. Let us introduce:

$$
\mathcal{J}:\left\{\begin{array}{ccc}
V & \rightarrow & V_{0} \times V_{1} \\
\psi & \mapsto & \left(\chi_{0, R} \psi, \chi_{1, R} \psi\right) .
\end{array}\right.
$$


As $\left(\chi_{0, R}, \chi_{1, R}\right)$ is a partition of the unity, $\mathcal{J}$ is injective. In particular, we notice that $\mathcal{J}: V \rightarrow \mathcal{J}(V)$ is bijective so that we have:

$$
\begin{aligned}
\lambda_{j} & =\inf _{\substack{F \subset \mathcal{J}(V) \\
\operatorname{dim} F=j}} \sup _{\psi \in \mathcal{J}^{-1}(F)} \frac{Q(\psi)}{\|\psi\|_{\widetilde{\Omega}}^{2}} \\
& =\inf _{\substack{F \subset \mathcal{J}(V) \\
\operatorname{dim} F=j}} \sup _{\psi \in \mathcal{J}^{-1}(F)} \frac{Q_{0, R}\left(\chi_{0, R} \psi\right)+Q_{1, R}\left(\chi_{1, R} \psi\right)}{\left\|\chi_{0, R} \psi\right\|_{\widetilde{\Omega}}^{2}+\left\|\chi_{1, R} \psi\right\|_{\widetilde{\Omega}}^{2}} \\
& =\inf _{\substack{F \subset \mathcal{J}(V) \\
\operatorname{dim} F=j}} \sup _{\left(\psi_{0}, \psi_{1}\right) \in F} \frac{Q_{0, R}\left(\psi_{0}\right)+Q_{1, R}\left(\psi_{1}\right)}{\left\|\psi_{0}\right\|_{\mathcal{O}_{0, R}}^{2}+\left\|\psi_{1}\right\|_{\mathcal{O}_{1, R}}^{2}} .
\end{aligned}
$$

As $\mathcal{J}(V) \subset V_{0} \times V_{1}$, we deduce by an application of Corollary 1.12:

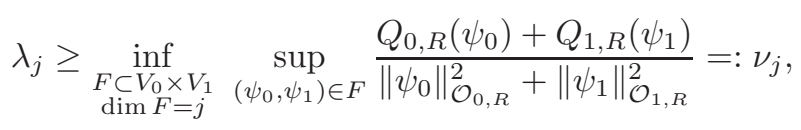

Let $A_{\ell, R}$ be the self-adjoint operator with domain $\operatorname{Dom}\left(A_{\ell, R}\right)$ associated with the coercive bilinear form corresponding to the quadratic form $Q_{\ell, R}$ on $V_{\ell}$. We see that $\nu_{j}$ in (21) is the $j$-th Rayleigh quotient of the diagonal self-adjoint operator $A_{R}$

$$
\left(\begin{array}{cc}
A_{0, R} & 0 \\
0 & A_{1, R}
\end{array}\right) \quad \text { with domain } \quad \operatorname{Dom}\left(A_{0, R}\right) \times \operatorname{Dom}\left(A_{1, R}\right) .
$$

The Rayleigh quotients of $A_{\ell, R}$ are associated with the quadratic form $Q_{\ell, R}$ for $\ell=0,1$. Thus $\nu_{j}$ is the $j$-th element of the ordered set

$$
\left\{\lambda_{k}\left(Q_{0, R}\right), k \geq 1\right\} \cup\left\{\lambda_{k}\left(Q_{1, R}\right), k \geq 1\right\} .
$$

Lemma 5.2 follows.

The operator $A_{0, R}$ is elliptic on a bounded open set, hence has a compact resolvent. Therefore we get:

Lemma 5.3. For all $R>0, \mathcal{N}\left(Q_{0, R}, 1\right)$ is finite.

To achieve the proof of Proposition 5.1, it remains to establish the following lemma:

Lemma 5.4. There exists $R_{0}>0$ such that, for $R \geq R_{0}, \mathcal{N}\left(Q_{1, R}, 1\right)$ is finite.

Proof. For all $\phi \in V_{1}$, we write:

where

$$
\phi=\Pi_{0} \phi+\Pi_{1} \phi
$$

$$
\Pi_{0} \phi(x, y)=\Phi(x) \sin y \quad \text { with } \quad \Phi(x)=\int_{0}^{\pi} \phi(x, y) \sin y \mathrm{~d} y
$$

is the projection on the first eigenvector of $-\partial_{y}^{2}$ on $\mathrm{H}_{0}^{1}(0, \pi)$, and $\Pi_{1}=\mathrm{Id}-\Pi_{0}$. We have, for all $\varepsilon>0$ :

$$
\begin{aligned}
Q_{1, R}(\phi) & =Q_{1, R}\left(\Pi_{0} \phi\right)+Q_{1, R}\left(\Pi_{1} \phi\right)-2 \int_{\mathcal{O}_{1, R}} R^{-2} W_{R}(x) \Pi_{0} \phi \Pi_{1} \phi \mathrm{d} x \mathrm{~d} y \\
& \geq Q_{1, R}\left(\Pi_{0} \phi\right)+Q_{1, R}\left(\Pi_{1} \phi\right)-\varepsilon^{-1} \int_{\mathcal{O}_{1, R}} R^{-2} W_{R}(x)\left|\Pi_{0} \phi\right|^{2} \mathrm{~d} x \mathrm{~d} y-\varepsilon \int_{\mathcal{O}_{1, R}} R^{-2} W_{R}(x)\left|\Pi_{1} \phi\right|^{2} \mathrm{~d} x \mathrm{~d} y .
\end{aligned}
$$

Since the second eigenvalue of $-\partial_{y}^{2}$ on $\mathrm{H}_{0}^{1}(0, \pi)$ is 4 , we have:

$$
\int_{\mathcal{O}_{1, R}}\left|\partial_{y} \Pi_{1} \phi\right|^{2} \mathrm{~d} x \mathrm{~d} y \geq 4\left\|\Pi_{1} \phi\right\|_{\mathcal{O}_{1, R}}^{2}
$$


Denoting by $M$ the maximum of $W_{R}$ (which is independent of $R$ ), and using (19) we deduce

$$
Q_{1, R}\left(\Pi_{1} \phi\right) \geq\left(4-M R^{-2}\right)\left\|\Pi_{1} \phi\right\|_{\mathcal{O}_{1, R}}^{2} .
$$

Combining this with (23) where we take $\varepsilon=1$, and with the definition (22) of $\Pi_{0}$, we find

$$
Q_{1, R}(\phi) \geq q_{R}(\Phi)+\left(4-2 M R^{-2}\right)\left\|\Pi_{1} \phi\right\|_{\mathcal{O}_{1, R}}^{2},
$$

where

$$
\begin{aligned}
q_{R}(\Phi) & =\int_{R}^{\infty} \tan ^{2} \theta\left|\partial_{x} \Phi\right|^{2}+|\Phi|^{2}-R^{-2} W_{R}(x)|\Phi|^{2} \mathrm{~d} x \\
& \geq \int_{R}^{\infty} \tan ^{2} \theta\left|\partial_{x} \Phi\right|^{2}+|\Phi|^{2}-R^{-2} M \mathbb{1}_{[R, 2 R]}|\Phi|^{2} \mathrm{~d} x .
\end{aligned}
$$

We choose $R=\sqrt{M}$ so that $\left(4-2 M R^{-2}\right)=2$, and then

$$
Q_{1, R}(\phi) \geq \tilde{q}_{R}(\Phi)+2\left\|\Pi_{1} \phi\right\|_{\mathcal{O}_{1, R}}^{2},
$$

where now

$$
\tilde{q}_{R}(\Phi)=\int_{R}^{\infty} \tan ^{2} \theta\left|\partial_{x} \Phi\right|^{2}+\left(1-\mathbb{1}_{[R, 2 R]}\right)|\Phi|^{2} \mathrm{~d} x .
$$

Let $\tilde{a}_{R}$ denote the 1D operator associated with the quadratic form $\tilde{q}_{R}$. From (24)-(25), we deduce that the $j$-th Rayleigh quotient of $A_{1, R}$ admits as lower bound the $j$-th Rayleigh quotient of the diagonal operator:

$$
\left(\begin{array}{cc}
\tilde{a}_{R} & 0 \\
0 & 2 \text { Id }
\end{array}\right)
$$

so that we find:

$$
\mathcal{N}\left(Q_{1, R}, 1\right) \leq \mathcal{N}\left(\tilde{q}_{R}, 1\right) .
$$

Finally, the eigenvalues $<1$ of $\tilde{a}_{R}$ can be computed explicitly and this is an elementary exercise to deduce that $\mathcal{N}\left(\tilde{q}_{R}, 1\right)$ is finite.

This concludes the proof of Proposition 5.1.

\section{Decay of Eigenvectors at infinity - Computations For large ANGLES}

\subsection{Decay at infinity}

In order to study theoretical properties of eigenvectors of the operator $\Delta_{\Omega_{\theta}}^{\text {Dir }}$ corresponding to eigenvalues below 1 , we use the equivalent configuration on $\widetilde{\Omega}_{\theta}$ introduced in section 3 , see also Figure 6 . The eigenvalues $<1$ of $\Delta_{\Omega_{\theta}}^{\text {Dir }}$ are the same as those of $\Delta_{\widetilde{\Omega}_{\theta}}^{\text {Mix }}$ (with Dirichlet conditions on the horizontal parts of the boundary of $\left.\widetilde{\Omega}_{\theta}\right)$ and the eigenvectors are isometric. The main result of this section is a quasi-optimal decay in the straight part $(0,+\infty) \times(0, \pi)$ of the set $\widetilde{\Omega}_{\theta}$ as $x \rightarrow \infty$.

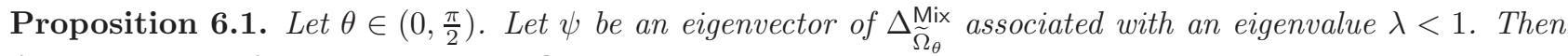
for all $\varepsilon>0$ the following integral is finite:

$$
\int_{0}^{\infty} \int_{0}^{\pi} \mathrm{e}^{2 \tilde{x}(\sqrt{1-\lambda}-\varepsilon)}\left(|\psi(\tilde{x}, \tilde{y})|^{2}+|\nabla \psi(\tilde{x}, \tilde{y})|^{2}\right) \mathrm{d} \tilde{y} \mathrm{~d} \tilde{x}<\infty .
$$




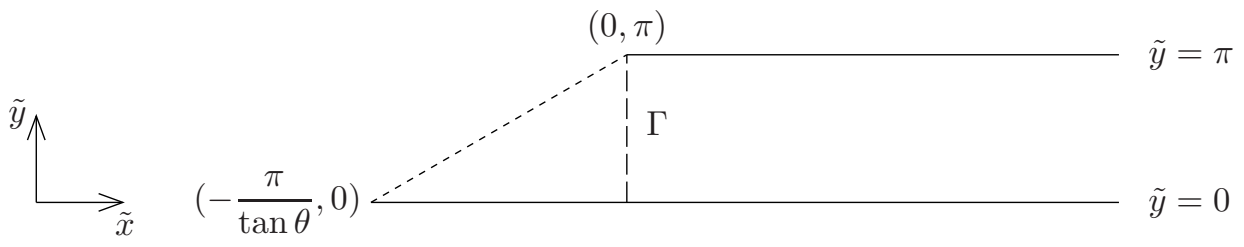

Figure 6 . The half-guide $\widetilde{\Omega}_{\theta}$ (here $\theta=\frac{\pi}{6}$ ).

Proof. We give here an elementary proof based on the representation of $\psi$ as solution of the Dirichlet problem in the half-strip $\Sigma:=\mathbb{R}_{+} \times(0, \pi)$

$$
\begin{cases}-\Delta \psi=\lambda \psi & \text { in } \Sigma, \\ \psi(\tilde{x}, 0)=0, \psi(\tilde{x}, \pi)=0 & \forall \tilde{x}>0, \\ \psi(0, \tilde{y})=g & \forall \tilde{y} \in(0, \pi)\end{cases}
$$

where $g$ is the trace of $\psi$ on the segment $\Gamma$, see Figure 6 . Since $\psi$ belongs to $\mathrm{H}^{1}(\Sigma)$, its trace on $\partial \Sigma$ belongs to $\mathrm{H}^{1 / 2}$. Because of the zero trace on the lines $\tilde{y}=0$ and $\tilde{y}=\pi$, we find that $g$ belongs to the smaller space ${ }^{2}$ $\mathrm{H}_{00}^{1 / 2}(\Gamma)$, which is the interpolation space of index $\frac{1}{2}$ between $\mathrm{H}_{0}^{1}(\Gamma)$ and $\mathrm{L}^{2}(\Gamma)$, cf. $[24]$.

We expand $\psi$ on the eigenvector basis of the operator $-\partial_{y}^{2}$, self-adjoint on $\mathrm{H}^{2} \cap \mathrm{H}_{0}^{1}(0, \pi)$. Its normalized eigenvectors are

We expand $g$ in this basis:

$$
v_{k}(\tilde{y})=\sqrt{\frac{2}{\pi}} \sin k \tilde{y} \quad \text { with eigenvalue } \quad \nu_{k}=k^{2} .
$$

$$
g(\tilde{y})=\sum_{k \geq 1} g_{k} v_{k}(\tilde{y}), \quad \text { where } g_{k}=\int_{0}^{\pi} g(\tilde{y}) v_{k}(\tilde{y}) \mathrm{d} \tilde{y} .
$$

Interpolating between (5) and (6), we find

$$
\|g\|_{\mathrm{H}_{00}^{1 / 2}(\Gamma)}^{2} \simeq \sum_{k \geq 1} k g_{k}^{2}
$$

We can easily solve (27) by separation of variables. We find

$$
\psi(\tilde{x}, \tilde{y})=\sum_{k \geq 1} \mathrm{e}^{-\tilde{x} \sqrt{k^{2}-\lambda}} g_{k} v_{k}(\tilde{y}) .
$$

The estimate of $\psi$ in (26) is then trivial. Let us prove now the estimate of $\partial_{\tilde{x}} \psi$. We use that $\sum_{k \geq 1} k g_{k}^{2}$ is finite so that we can write:

$$
\partial_{\tilde{x}} \psi(\tilde{x}, \tilde{y})=-\sum_{k \geq 1} \sqrt{k^{2}-\lambda} \mathrm{e}^{-\tilde{x} \sqrt{k^{2}-\lambda}} g_{k} v_{k}(\tilde{y}) .
$$

We have:

$$
\mathrm{e}^{\tilde{x}(\sqrt{1-\lambda}-\varepsilon)} \partial_{\tilde{x}} \psi(\tilde{x}, \tilde{y})=-\sum_{k \geq 1} \sqrt{k^{2}-\lambda} \mathrm{e}^{\tilde{x}\left(\sqrt{1-\lambda}-\sqrt{k^{2}-\lambda}\right)} \mathrm{e}^{-\varepsilon \tilde{x}} g_{k} v_{k}(\tilde{y})
$$

\footnotetext{
${ }^{2}$ The space $\mathrm{H}_{00}^{1 / 2}(\Gamma)$ is the subspace of $\mathrm{H}^{1 / 2}(\Gamma)$ spanned by the functions $v$ such that
}

$$
\int_{0}^{\pi} \frac{v^{2}(\tilde{y})}{\tilde{y}(\pi-\tilde{y})} \mathrm{d} \tilde{y}<\infty .
$$


leading to the $\mathrm{L}^{2}$ estimate:

$$
\begin{aligned}
\int_{0}^{\infty} \int_{0}^{\pi}\left|\mathrm{e}^{\tilde{x}(\sqrt{1-\lambda}-\varepsilon)} \partial_{\tilde{x}} \psi(\tilde{x}, \tilde{y})\right|^{2} \mathrm{~d} \tilde{y} \mathrm{~d} \tilde{x} & =\sum_{k \geq 1} \int_{0}^{\infty}\left(k^{2}-\lambda\right) \mathrm{e}^{2 \tilde{x}\left(\sqrt{1-\lambda}-\sqrt{k^{2}-\lambda}\right)} \mathrm{e}^{-2 \varepsilon \tilde{x}} g_{k}^{2} \mathrm{~d} \tilde{x} \\
& \leq\left(\sum_{k \geq 1} k g_{k}^{2}\right) \sup _{k \geq 1} \int_{0}^{\infty} k \mathrm{e}^{-2 \gamma \tilde{x} \sqrt{k^{2}-1}} \mathrm{e}^{-2 \varepsilon \tilde{x}} \mathrm{~d} \tilde{x}
\end{aligned}
$$

where $\gamma=\gamma(\lambda)>0$ is a constant, uniform with respect to $k \geq 1$. Using the change of variables $\tilde{x} \mapsto \sqrt{k} \tilde{x}$, we can see that the integrals

$$
\int_{0}^{+\infty} k \mathrm{e}^{-2 \tilde{x}\left(\varepsilon+\gamma \sqrt{k^{2}-1}\right)}
$$

are uniformly bounded as $k \rightarrow \infty$, which ends the proof of the estimate of $\partial_{\tilde{x}} \psi$ in (26). The estimate of $\partial_{\tilde{y}} \psi$ is similar.

Remark 6.2. Under the conditions of Proposition 6.1, we have also a sharp global $\mathrm{L}^{\infty}$ estimate

$$
\left\|\mathrm{e}^{\tilde{x} \sqrt{1-\lambda}} \psi\right\|_{L^{\infty}\left(\widetilde{\Omega}_{\theta}\right)}<\infty
$$

To prove this we use again the representation $(28)$ and the fact that the trace $g$ is more regular than $\mathrm{H}_{00}^{1 / 2}(\Gamma)$. In fact, as a consequence of the characterization (10) of the domain of the operator $\Delta_{\Omega_{\theta}}^{\text {Dir }}$, the trace $g$ belongs to $\mathrm{H}_{0}^{1}(\Gamma)$ and, therefore, the sum $\sum_{k \geq 1} k^{2} g_{k}^{2}$ is finite by (6). Then, we have:

$$
\mathrm{e}^{\tilde{x} \sqrt{1-\lambda}} \psi(\tilde{x}, \tilde{y})=\sum_{k \geq 1} \mathrm{e}^{\tilde{x}\left(\sqrt{1-\lambda}-\sqrt{k^{2}-\lambda}\right)} g_{k} v_{k}(\tilde{y}) .
$$

Taking absolute values, we get:

$$
\mathrm{e}^{\tilde{x} \sqrt{1-\lambda}}|\psi(\tilde{x}, \tilde{y})| \leq \sqrt{\frac{2}{\pi}} \sum_{k \geq 1}\left|g_{k}\right| \leq \sqrt{\frac{2}{\pi}}\left(\sum_{k \geq 1} k^{-2}\right)^{1 / 2}\left(\sum_{k \geq 1} k^{2}\left|g_{k}\right|^{2}\right)^{1 / 2} .
$$

Remark 6.3. The estimate (26) can also be proved with a general method due to Agmon (see for instance [1]).

\subsection{Computations for large angles}

When $\theta \rightarrow \frac{\pi}{2}, \lambda_{1}(\theta)$ tends to 1 , see [2]. The representation (28) shows that in such a situation, the behavior of the associated eigenvector $\psi$ is dominated by its first term, proportional to

$$
\mathrm{e}^{-\tilde{x} \sqrt{1-\lambda}} \sin (\tilde{y})
$$

In order to compute such an eigenpair by a finite element method, we have to be careful and take large enough domains - we simply put Dirichlet conditions on an artificial boundary far enough from the corners of the guide. Our computations are performed in the model half-guide $\Omega:=\Omega_{\pi / 4}^{+}$for the scaled operator

$$
\mathscr{L}_{\theta}:=-2 \sin ^{2} \theta \partial_{u}^{2}-2 \cos ^{2} \theta \partial_{v}^{2}
$$

equivalent to $-\Delta$ in $\Omega_{\theta}^{+}$through the variable change

$$
u=x_{1} \sqrt{2} \sin \theta \text { and } \quad v=x_{2} \sqrt{2} \cos \theta .
$$




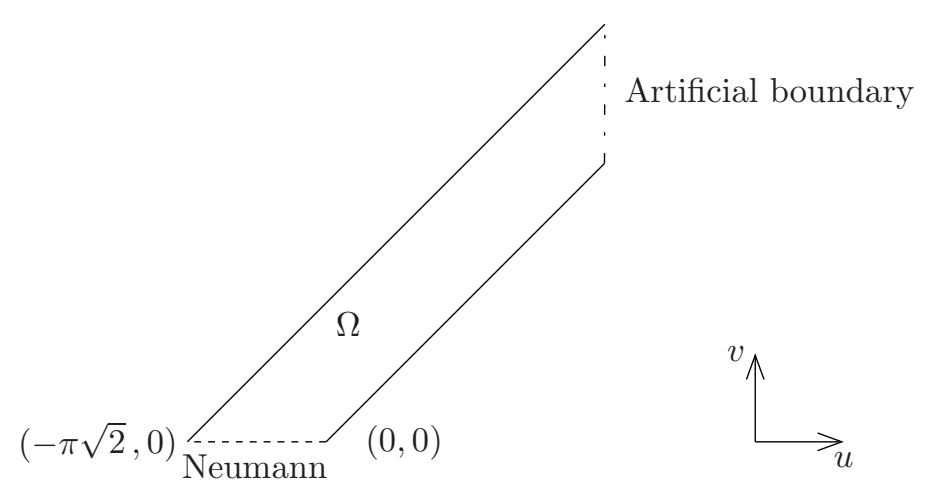

Figure 7 . The model half-guide $\Omega:=\Omega_{\pi / 4}^{+}$.

We use a Galerkin discretization by finite elements in a truncated subset of $\Omega$ with Dirichlet condition on the artificial boundary, see Figure 7. According to Corollary 1.12, cf. also Examples 1.13 and 1.14, the eigenvalues $\lambda_{j}^{\text {cpt }}(\theta)$ of the discretized problem are larger than the Rayleigh quotients $\lambda_{j}(\theta)$ of $\mathscr{L}_{\theta}$. When the discretization gets finer and the computational domain, larger, $\lambda_{j}^{\mathrm{cpt}}(\theta)$ tends to $\lambda_{j}(\theta)$ for $j=1,2, \ldots$

For the values of $\theta$ considered in this section $\left(\theta \geq \frac{\pi}{4}\right)$, the numerical evidence is that the discrete spectrum of $\mathscr{L}_{\theta}$ has only one element $\lambda_{1}(\theta)$. The numerical effect of this is the convergence to 1 of all other computational eigenvalues $\lambda_{j}^{\text {cpt }}(\theta)$ for $j=2,3, \ldots$

The computations represented in Figure 8 are performed with the artificial boundary set at the abscissa $u=5 \pi \sqrt{2}$. The plots are mapped back to the corresponding physical domain by a postprocessing of the numerical results.

The computations in Figure 9 are performed with the artificial boundary set at the abscissa $u=10 \pi \sqrt{2}$. The plots use the computational domain because the corresponding physical domains would be too much elongated to be represented.

\section{Accumulation of eigenpairs for small angles}

When $\theta$ tends to 0 , there is more and more room for eigenvectors between the two corners of the guide.

For any rectangular box $\mathcal{B}$ contained in $\Omega_{\theta}$ like in Figure 10, by the monotonicity of Dirichlet eigenvalues (Example 1.13), we know that for any $j$

$$
\lambda_{j}(\theta) \leq \lambda_{j}(\mathcal{B})
$$

where $\lambda_{j}(\theta)$ are the Rayleigh quotients of $\Delta_{\Omega_{\theta}}^{\text {Dir }}$ and $\lambda_{j}(\mathcal{B})$ the Dirichlet eigenvalues on $\mathcal{B}$. We choose $\mathcal{B}$ in the form of a rectangle bounded by the vertical lines $x_{1}=-\alpha \pi$ and $x_{1}=0$, and the horizontal lines $x_{2}= \pm \beta \pi$. Thus $\alpha$ and $\beta$ satisfy, $c f$. (8)

$$
\alpha \in\left(0, \frac{1}{\sin \theta}\right), \quad \beta \pi=(-\alpha \sin \theta+1) \frac{\pi}{\cos \theta} .
$$

The eigenvalues of the Dirichlet problem in $\mathcal{B}$ are

$$
\frac{k^{2}}{4 \beta^{2}}+\frac{\ell^{2}}{\alpha^{2}}, \quad k, \ell \in\{1,2, \ldots\}
$$




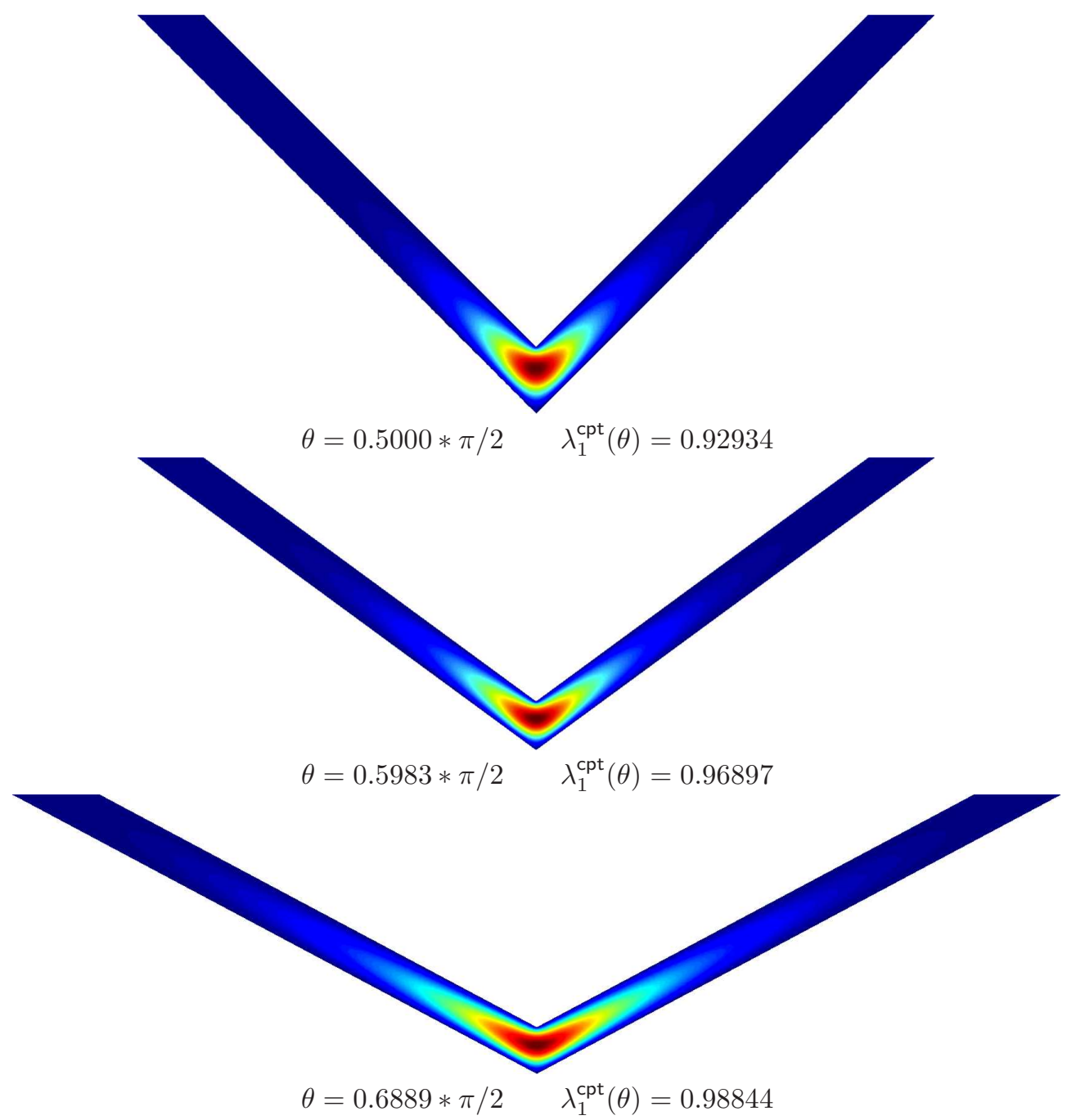

Figure 8. Computations for moderately large angles. Plots of the first eigenvectors in the physical domain (rotated by $\frac{\pi}{2}$ ). Numerical value of the corresponding eigenvalue $\lambda_{1}(\theta)$.

We look for the eigenvalues $\lambda_{j}(\mathcal{B})$ less than 1 . Therefore $\alpha$ has to be chosen $>1$. Thus $\beta<(1-\sin \theta) / \cos \theta \leq 1$ for any $\theta$. As a consequence $k=1$ and the eigenvalues $\lambda_{j}(\mathcal{B})$ less than 1 are necessarily of the form

$$
\begin{aligned}
\lambda_{j}(\mathcal{B}) & =\frac{1}{4 \beta^{2}}+\frac{j^{2}}{\alpha^{2}} \\
& =\frac{\cos ^{2} \theta}{4(1-\alpha \sin \theta)^{2}}+\frac{j^{2}}{\alpha^{2}} .
\end{aligned}
$$

We optimize $\mathcal{B}$ : The minimum of $\lambda_{j}(\mathcal{B})$ is obtained for $\alpha$ such that

$$
\frac{\sin \theta \cos ^{2} \theta}{2(1-\alpha \sin \theta)^{3}}-\frac{2 j^{2}}{\alpha^{3}}=0
$$



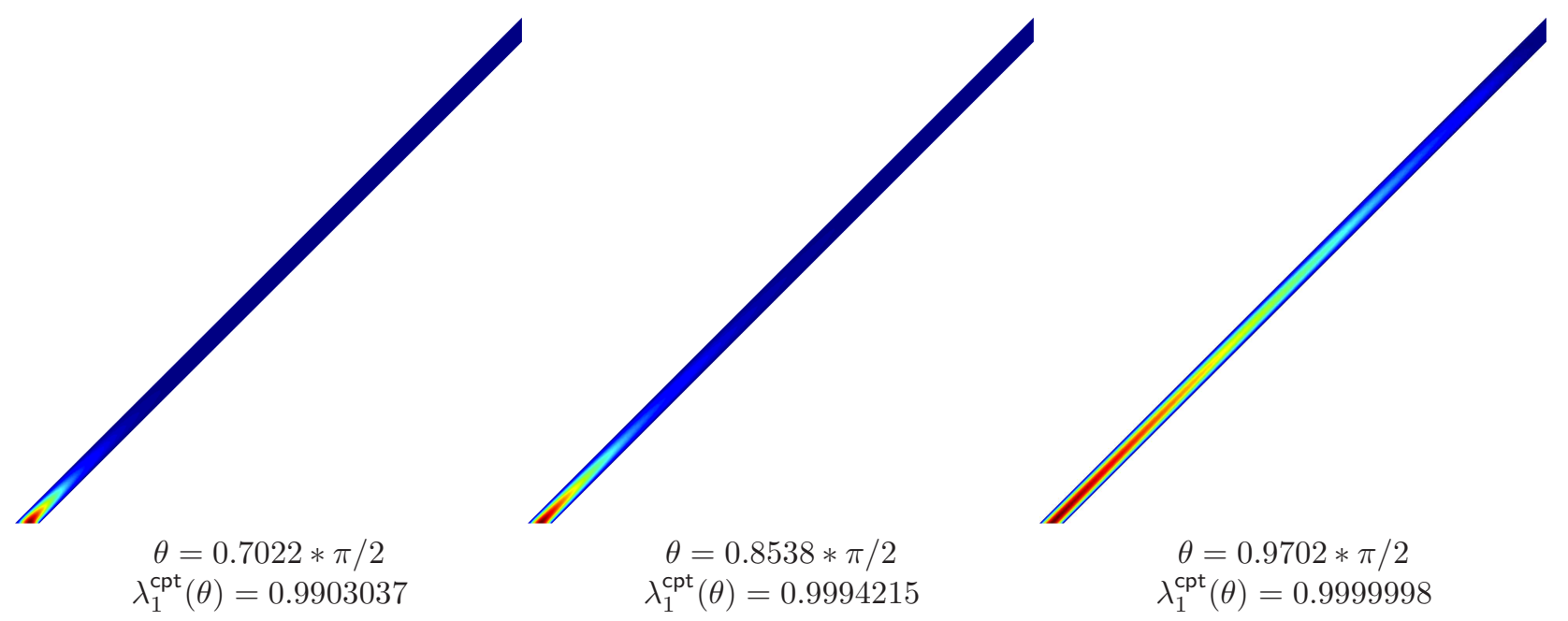

Figure 9. Computations for very large angles with the mesh M4L (see Figure 17). Plots in the computational domain $\Omega$.
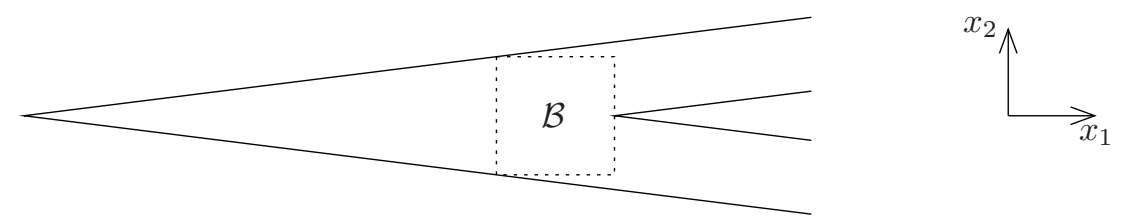

Figure 10. The waveguide $\Omega_{\theta}$ and a Dirichlet box $\mathcal{B}$ inside.

Since we are interested in the behavior as $\theta \rightarrow 0$, we take without asymptotic loss

$$
\alpha=4^{1 / 3} j^{2 / 3} \sin ^{-1 / 3} \theta
$$

which provides

$$
\lambda_{j}(\mathcal{B})=\frac{1}{4}\left(\frac{\cos ^{2} \theta}{\left(1-4^{1 / 3} j^{2 / 3} \sin ^{2 / 3} \theta\right)^{2}}+4^{1 / 3} j^{2 / 3} \sin ^{2 / 3} \theta\right)
$$

As a consequence, as soon as the quantity $Z:=4^{1 / 3} j^{2 / 3} \sin ^{2 / 3} \theta$ is less that the first root of the equation

$$
\frac{1}{4}\left(\frac{1}{(1-Z)^{2}}+Z\right)=1
$$

i.e. for $Z \leq 0.4679$, we have $\lambda_{j}(\mathcal{B})<1$ and, hence, $\lambda_{j}(\theta)<1$. This implies that the maximal number $J$ such that $\lambda_{J}(\mathcal{B})<1$ is greater than

$$
0.4679^{3 / 2} \cdot 0.5 \sin ^{-1} \theta \simeq 0.1601 \sin ^{-1} \theta .
$$

Therefore the number of eigenvalues of $\Delta_{\Omega_{\theta}}^{\text {Dir }}$ less than 1 tends to infinity (at least) like $\theta^{-1}$ as $\theta$ tends to 0 .

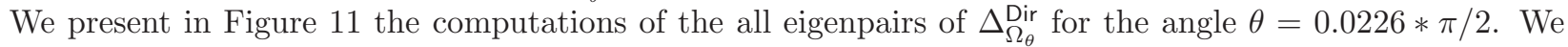
find 10 eigenvalues $<1$. Note that for this angle $\theta$, the numerical value of $0.1601 \sin ^{-1} \theta$ is 4.5103 . 


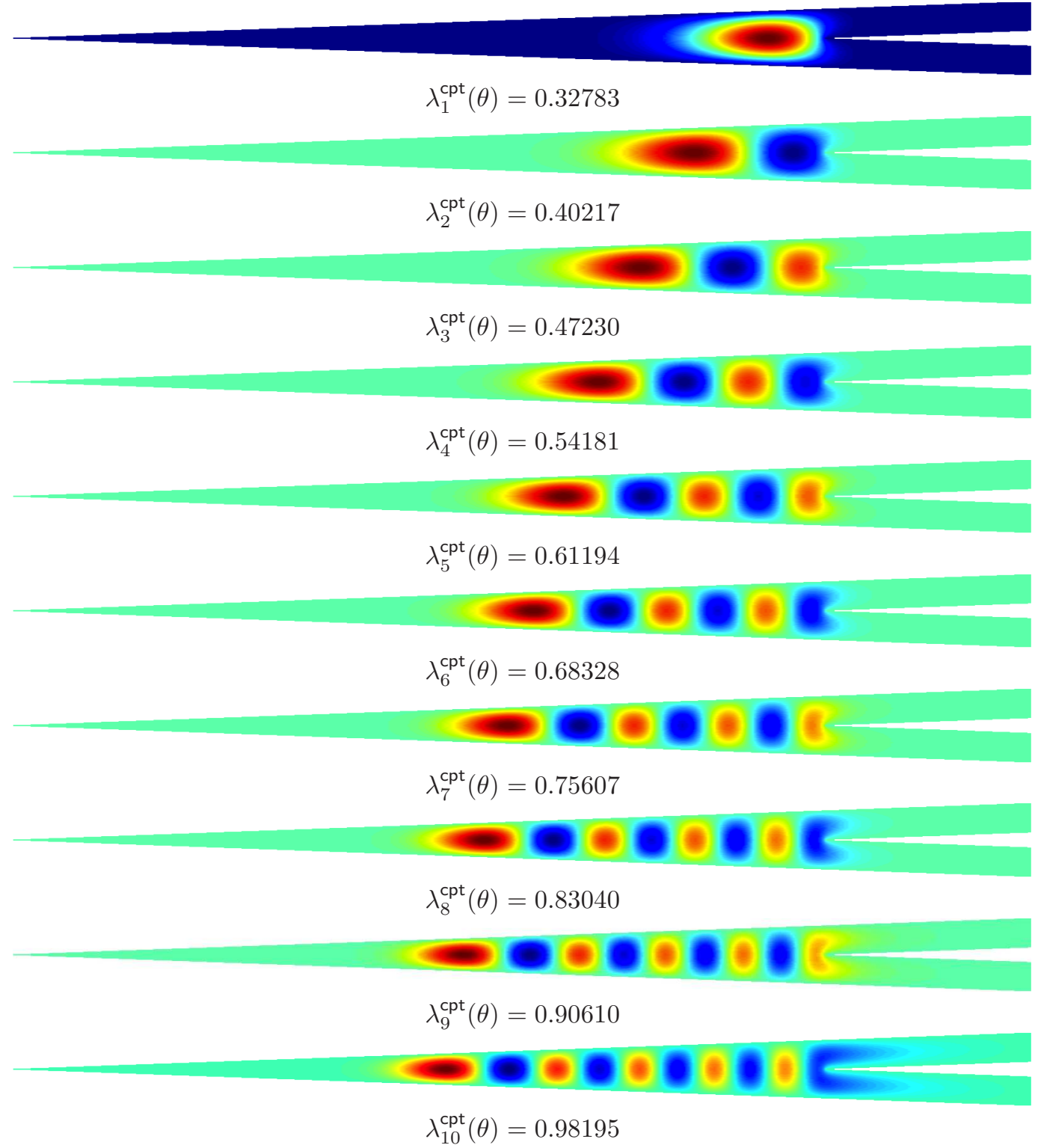

Figure 11. Computations for $\theta=0.0226 * \pi / 2 \sim 2^{\circ}$ with the mesh M64S (see Figure 18). Numerical values of the 10 eigenvalues $\lambda_{j}(\theta)<1$. Plots of the associated eigenvectors in the physical domain.

\section{Asymptotic BeHAVIOR OF EIGENPAIRS FOR SMALL ANGLES}

We present in Figures 12 and 13 computations of the first eigenvector for smaller and smaller values of the angle $\theta$. We notice that the eigenvectors look similar, with the appearance of a short scale in the horizontal variable.

We perform in [9] asymptotic expansions of the first eigenpairs at any order as $\theta \rightarrow 0$, using techniques of semi-classical analysis (see for instance $[19,20]$ and also [26]). We briefly describe now some of the results proved there. 

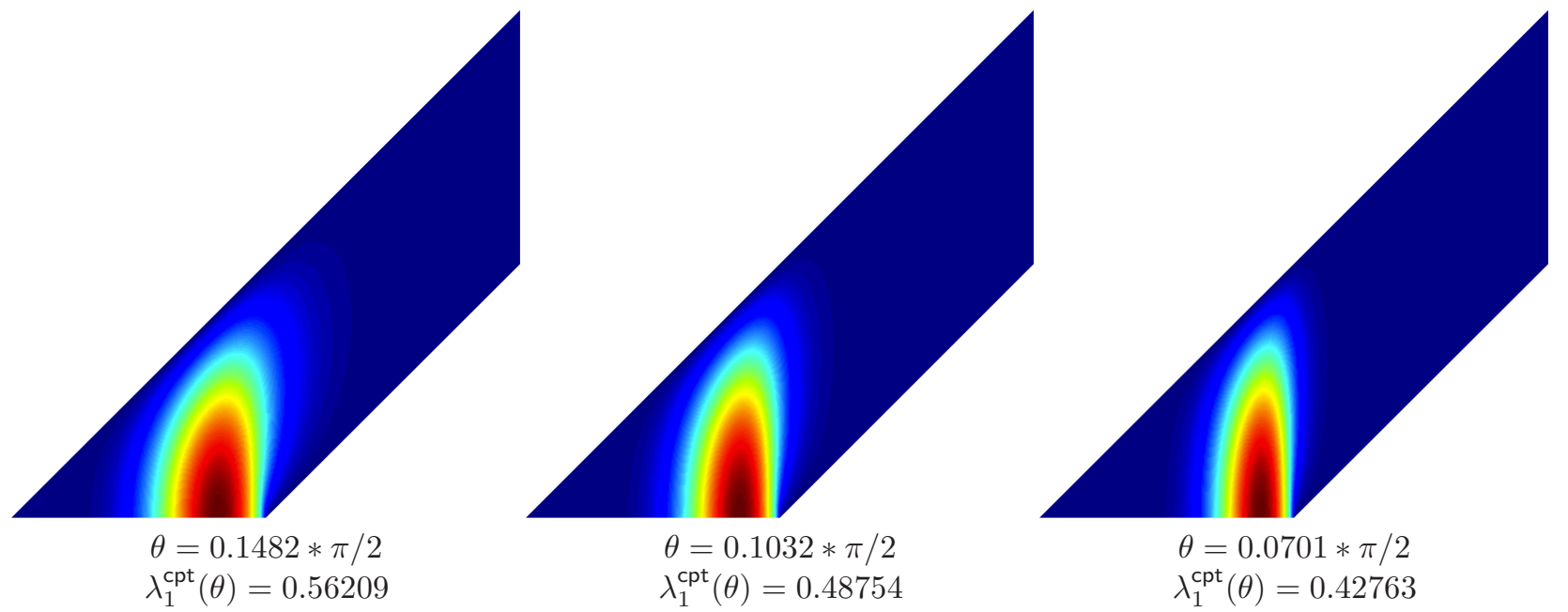

Figure 12. Computations for small angles. Plots in the computational domain $\Omega$.
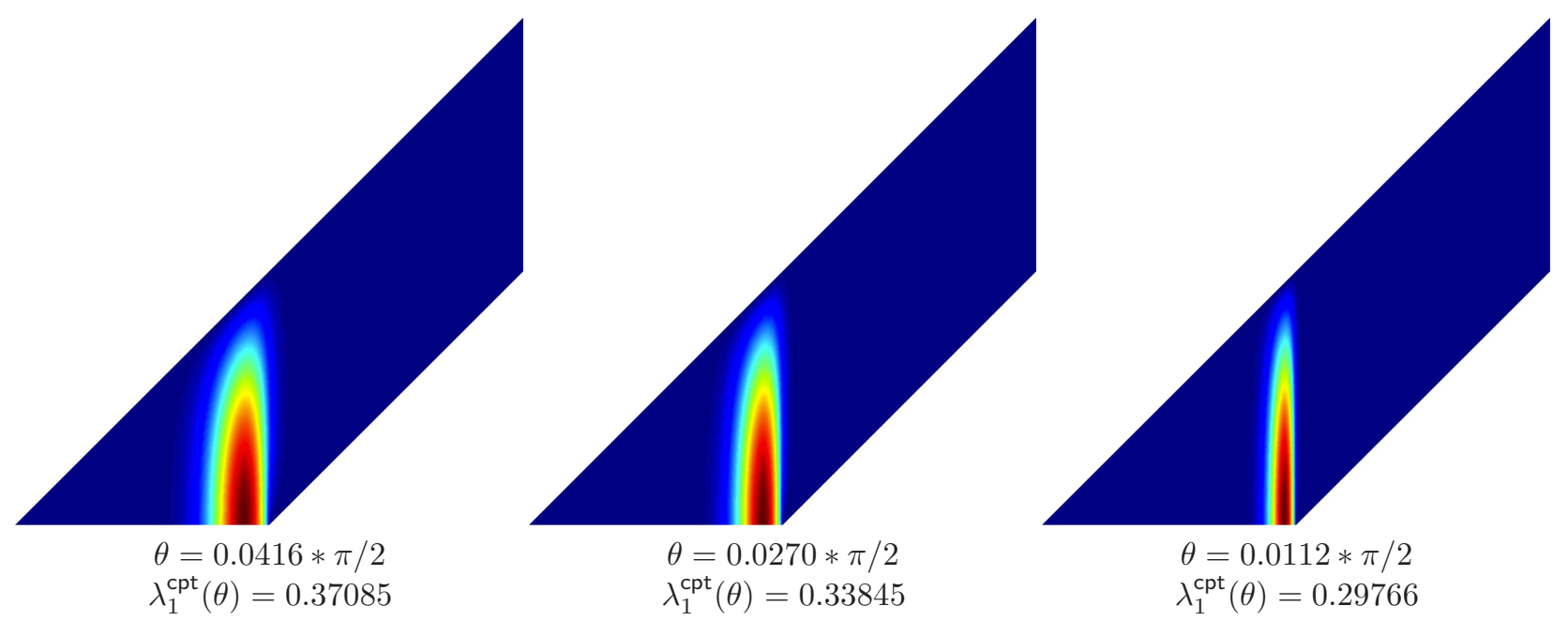

Figure 13. Computations for very small angles. Plots in the computational domain $\Omega$.

Let us recall that the eigenvalues $\lambda_{j}(\theta)<1$ of our operator $\Delta_{\Omega_{\theta}}^{\text {Dir }}$ coincide with those of the scaled operator

$$
\mathscr{L}_{\theta}:=-2 \sin ^{2} \theta \partial_{u}^{2}-2 \cos ^{2} \theta \partial_{v}^{2}
$$

in the model half-guide $\Omega$. The construction and validation of asymptotic expansions for the eigenpairs of $\mathscr{L}_{\theta}$ rely on a Born-Oppenheimer approximation. Roughly, this consists of four steps:

(1) Definition of an operator $\mathscr{L}_{\theta}^{\mathrm{BO}}$ in the horizontal variable $u$ by replacing the operator in the vertical variable $\mathscr{N}_{u}:=-2 \cos ^{2} \theta \partial_{v}^{2}$ in each slice $u=$ const. by its first eigenvalue $\Lambda(u)$

(2) Semi-classical analysis of the eigenpairs of $\mathscr{L}_{\theta}^{\mathrm{BO}}$ as $\theta \rightarrow 0$.

(3) Determination of a change of variables $(u, v) \mapsto(u, t)$ on $\Omega$ in order to exhibit a tensor product structure. Here appears the role of the limit operator $\mathscr{N}_{0}$ as $u \nearrow 0$. Its first eigenvector is $v \mapsto \cos (v / 2)$.

(4) Construction of expansions of eigenvectors in the new variables. 


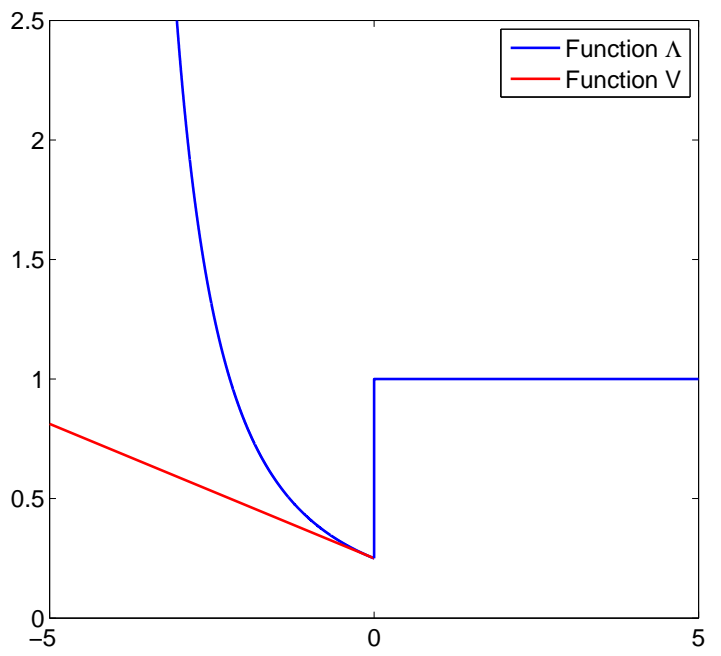

Figure 14. The Born-Oppenheimer potential $\Lambda$ and its left tangent at $u=0$ (here $\cos ^{2} \theta$ is set to 1$)$.

STEP 1: Definition of $\mathscr{L}_{\theta}^{\text {BO }}$.

Let $\mathcal{I}(u)$ be the intersection of $\Omega$ with the vertical line of abscissa $u$.

- For $u \in(-\pi \sqrt{2}, 0), \mathcal{I}(u)=(0, u+\pi \sqrt{2})$. The operator $\mathscr{N}_{u}$ has Neumann condition at 0 and Dirichlet at $u+\pi \sqrt{2}$. Thus

$$
\Lambda(u)=2 \cos ^{2} \theta \frac{\pi^{2}}{4(u+\pi \sqrt{2})^{2}}
$$

- For $u \in(0,+\infty), \mathcal{I}(u)=(u, u+\pi \sqrt{2})$. The operator $\mathscr{N}_{u}$ has Dirichlet conditions at both ends. Thus

$$
\Lambda(u)=\cos ^{2} \theta
$$

The Born-Oppenheimer operator is

$$
\mathscr{L}_{\theta}^{\mathrm{BO}}=-2 \sin ^{2} \theta \partial_{u}^{2}+\Lambda(u)
$$

STEP 2: Semi-classical analysis of $\mathscr{L}_{\theta}^{\mathrm{BO}}$.

The operator $\mathscr{L}_{\theta}^{\mathrm{BO}}$ can be viewed as a $1 \mathrm{D}$ Schrödinger operator with potential $\Lambda$. The potential has a well with bottom at $u=0$. The well is not smooth and has a triangular shape, see Figure 14.

The behavior of the eigenpairs of $\mathscr{L}_{\theta}^{\mathrm{BO}}$ as $\theta \rightarrow 0$ is governed by the Taylor expansion of the potential $\Lambda$ at the well bottom $u=0$, i.e. by the tangent potential $V$ defined by

$$
V(u)=\cos ^{2} \theta \begin{cases}\frac{1}{4}-\frac{u}{2 \pi \sqrt{2}}, & \text { if } u<0 \\ 1, & \text { if } u>0\end{cases}
$$

The corresponding model problem is the problem of the behavior as $h \rightarrow 0$ of the eigenpairs of the operator

$$
\mathscr{H}_{h}=-h^{2} \partial_{u}^{2}+ \begin{cases}-u, & \text { if } u<0 \\ 1, & \text { if } u>0\end{cases}
$$


The potential barrier on $(0,+\infty)$ produces a Dirichlet condition at $u=0$ for the leading terms of the asymptotics: We are led to the Airy-type eigenvalue problem

$$
-h^{2} \partial_{u}^{2} \psi_{h}-u \psi_{h}=E_{h} \psi_{h} \text { on }(-\infty, 0), \quad \text { with } \quad \psi(0)=0 .
$$

The change of variables $u \mapsto X=u h^{-2 / 3}$ transforms the above equation into the reverse Airy equation

$$
-\partial_{X}^{2} \Psi-X \Psi=\mu \Psi \quad \text { on }(0,+\infty), \quad \text { with } \quad \Psi(0)=0, \quad\left(\text { where } \mu=h^{-2 / 3} E_{h}\right)
$$

whose solutions can be easily exhibited: As we will see, the eigenvalues $\mu$ are the zeros of the reverse Airy function $\mathrm{A}(X):=\mathrm{Ai}(-X)$, where $\mathrm{Ai}$ is the standard Airy function. The zeros of $\mathrm{A}$ form an increasing sequence of positive numbers, which we denote by $z_{\mathrm{A}}(j), j \geq 1$.

Since $-\mathrm{A}^{\prime \prime}(X)-X \mathrm{~A}(X)=0$ we have for any $E \in \mathbb{R}$

$$
-\mathrm{A}^{\prime \prime}(X)-(X-E) \mathrm{A}(X)=E \mathrm{~A}(X) \quad \text { i.e. } \quad-\mathrm{A}^{\prime \prime}(X+E)-X \mathrm{~A}(X+E)=E \mathrm{~A}(X+E) .
$$

If $E=z_{\mathrm{A}}(j)$, then $\mathrm{A}(X+E)$ vanishes at $X=0$, hence

$$
\left(z_{\mathrm{A}}(j), \mathrm{A}\left(X+z_{\mathrm{A}}(j)\right)\right) \text { is an eigenpair. }
$$

Conversely, all eigenpairs are of this form.

We deduce that the eigenvalues of problem (30) are $E_{h}=h^{2 / 3} z_{\mathrm{A}}(j)$ and the associated eigenvectors are $\psi(u)=\mathrm{A}\left(u h^{-2 / 3}+z_{\mathrm{A}}(j)\right)$. form

Coming back to our operator $\mathscr{L}_{\theta}^{\mathrm{BO}}$, we prove in [9] that its eigenvalues have asymptotic expansions of the

$$
\lambda_{j}^{\mathrm{BO}}(\theta) \underset{\theta \rightarrow 0}{\simeq} \frac{1}{4}+\frac{2 \theta^{2 / 3} z_{\mathrm{A}}(j)}{(4 \pi \sqrt{2})^{2 / 3}}+\sum_{n \geq 3} \theta^{n / 3} \gamma_{j, n}^{\mathrm{BO}},
$$

where $\gamma_{j, n}^{\text {BO }}$ are some real coefficients. The eigenvectors have expansions in powers of $\theta^{1 / 3}$, using the scale $u h^{-2 / 3}$ for $u<0$ and $u h^{-1}$ for $u>0$.

STEP 3: Tensorial structure of $\mathscr{L}_{\theta}$.

On the left part of $\Omega$, i.e. its triangular part Tri in the half-plane $u<0$, we perform a change of variables to transform Tri into a square:

$$
\text { Tri } \ni(u, v) \mapsto(u, t) \in(-\pi \sqrt{2}, 0) \times(0, \pi \sqrt{2}), \quad \text { with } \quad t=\frac{v \pi \sqrt{2}}{u+\pi \sqrt{2}} .
$$

On the right part of $\Omega$, i.e. its strip part contained in the half-plane $u>0$, we perform a change of variables to transform it into a horizontal strip:

$$
(u, v) \mapsto(u, \tau) \in(0,+\infty) \times(0, \pi \sqrt{2}), \quad \text { with } \quad \tau=v-u .
$$

This allows to work with the Taylor expansions of the transformed operator around $u=0$, on the left and on the right. The operator $\mathscr{N}_{0-}=-2 \partial_{t}^{2}$ appears on the left with Dirichlet condition at $t=1$ and Neumann at $t=0$. Its first eigenvector is $\cos \frac{t}{2}$, associated with the eigenvalue $\frac{1}{4}$. The operator $\mathscr{N}_{0+}=-2 \partial_{\tau}^{2}$ appears on the right with Dirichlet condition at $t=0,1$.

STEP 4: Asymptotics of the eigenpairs of $\mathscr{L}_{\theta}$.

The projection on the eigenvector $t \mapsto \cos \frac{t}{2}$ appears naturally at the first step of the expansion (this projection is sometimes called Feshbach or Grushin projection) and lets appear $\mathscr{L}_{\theta}^{\mathrm{BO}}$. A complete asymptotics for eigenpairs 

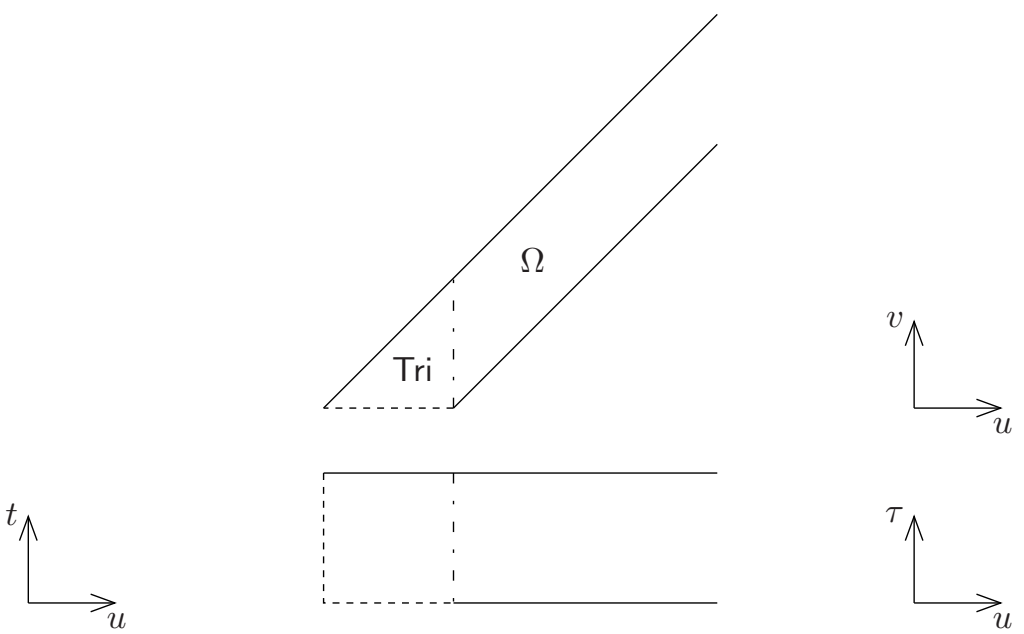

FiguRE 15 . The model waveguide $\Omega$ and the change of variables.

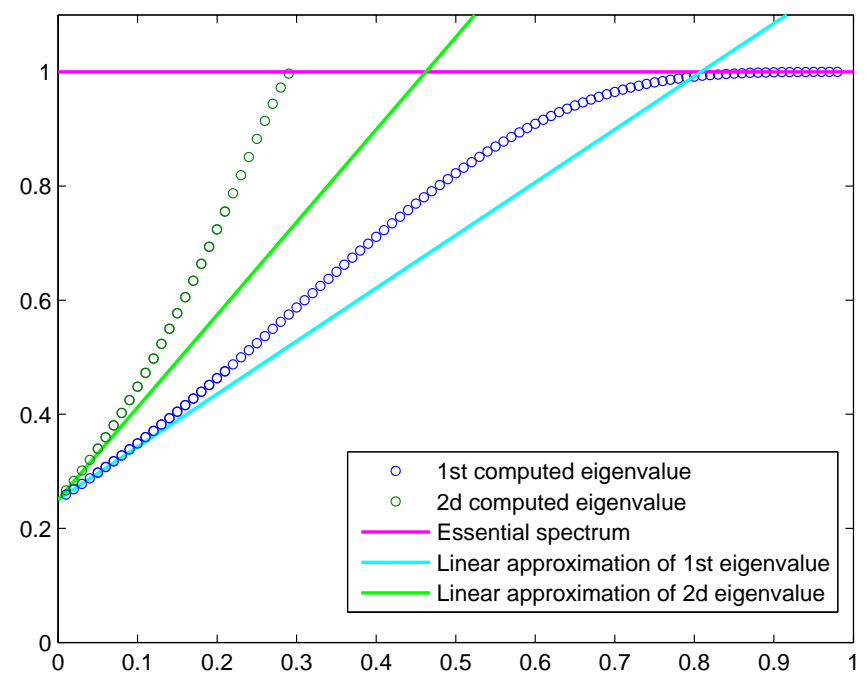

Figure 16. The eigenvalues $\lambda_{1}$ and $\lambda_{2}$ as functions of $(\theta * 2 / \pi)^{2 / 3}$.

can be constructed, and in a further step, validated. As a result $\lambda_{j}(\theta)$ has an expansion similar to $\lambda_{j}^{\mathrm{BO}}(\theta)$, with different coefficients for $n \geq 3$ :

$$
\lambda_{j}(\theta) \underset{\theta \rightarrow 0}{\simeq} \frac{1}{4}+\frac{2 \theta^{2 / 3} z_{\mathrm{A}}(j)}{(4 \pi \sqrt{2})^{2 / 3}}+\sum_{n \geq 3} \theta^{n / 3} \gamma_{j, n}
$$

where $\gamma_{j, n}$ are some real coefficients. In Figure 16 we display the functions $(\theta * 2 / \pi)^{2 / 3} \mapsto \lambda_{j}(\theta)$ for $j=$ 1,2 (computed by finite elements) and their linear approximation as $\theta \rightarrow 0$, corresponding to the two-term approximation in $(34)$, i.e. $(\theta * 2 / \pi)^{2 / 3} \mapsto \frac{1}{4}+\frac{2 \theta^{2 / 3} z_{\mathrm{A}}(j)}{(4 \pi \sqrt{2})^{2 / 3}}$.

In the reference domain $\Omega$, the eigenvectors of $\mathscr{L}_{\theta}$ have a multiscale expansion in powers of $\theta^{1 / 3}$. On the left side, there are two scales, the ultra-short scale $\left(u \theta^{-1}, v\right)$ and the short scale $\left(u \theta^{-2 / 3}, v\right)$, on the right side there 
is only the ultra-short scale. The asymptotics is dominated by its first term, which is

$$
\mathrm{A}\left(\frac{u}{\theta^{2 / 3}}-z_{\mathrm{A}}(1)\right) \cos \frac{t}{2} .
$$

This structure explains the results seen in Figures 12-13.

\section{Convergence of the finite element method}

We now present some aspects of the computation process that led to the numerical results shown in the previous sections.

As described in the section 6.2, the eigenvalue problem writes $\mathscr{L}_{\theta} \psi=\lambda \psi$ in the domain $\Omega$ with homogeneous Dirichlet conditions on its boundary, except on the horizontal segment where Neumann conditions are set, see identity (29) and Figure 7. The associate bilinear form is

$$
b\left(\psi, \psi^{\prime}\right)=\int_{\Omega} 2 \sin ^{2} \theta\left(\partial_{u} \psi \partial_{u} \psi^{\prime}\right)+2 \cos ^{2} \theta\left(\partial_{v} \psi \partial_{v} \psi^{\prime}\right) \mathrm{d} u \mathrm{~d} v
$$

defined on the corresponding form domain

$$
V=\left\{\psi \in \mathrm{H}^{1}(\Omega): \psi=0 \text { on } \partial_{\mathrm{Dir}} \Omega\right\} .
$$

The eigenvalue problem writes in variational form: find non-zero $\psi \in V$ and $\lambda$ such that

$$
\forall \psi^{\prime} \in V, \quad b\left(\psi, \psi^{\prime}\right)=\lambda\left\langle\psi, \psi^{\prime}\right\rangle_{\mathrm{L}^{2}(\Omega)} .
$$

By Galerkin projection on a finite dimension subspace $V_{\mathrm{fds}}$ of $V$, this problem can be rewritten as the generalized eigenvalue problem: find the eigenpairs $(\lambda, w)$ such that $S w=\lambda M w$, where $S$ and $M$ are the stiffness and mass matrices associated with a basis $\left(\Psi_{1}, \ldots, \Psi_{N}\right)$ of $V_{\mathrm{fds}}$ :

$$
S=\left(b\left(\Psi_{j}, \Psi_{k}\right)\right)_{1 \leq j, k \leq N} \text { and } \quad M=\left(\left\langle\Psi_{j}, \Psi_{k}\right\rangle_{\mathrm{L}^{2}(\Omega)}\right)_{1 \leq j, k \leq N} .
$$

The computation process consists of two main steps: first, using a finite element method that leads to the two matrices $S$ and $M$, and second, using an algorithm to compute the eigenpairs. In the following two sections, we focus on the algorithm for the computation of the eigenpairs and then on the influence of the choice of meshes and polynomial degrees in the finite element method.

All the computations have been done in double precision arithmetic, on a iMac computer (4 GB memory, 3.06 GHz Intel Core 2 Duo processor).

\subsection{Computation of the eigenpairs}

We have first written a program using the Fortran 77 finite element library "Melina" [25], which also provides a routine to compute eigenpairs of real symmetric eigenvalue problems. This routine uses an algorithm based on subspaces iterations. We call Mel this method.

We have also written another program using the $\mathrm{C}++$ version of the previous library, called "Melina++". The computation of the eigenpairs have been made using the well-known package ARPACK $++[17]$. We call Arp this method. We thus were able to reproduce the results obtained with the Mel method, which is a cross validation of both methods.

Here, we compare the computation of the eigenpairs with the two methods. For this purpose, in both cases, we fix the parameters governing the finite element part: interpolation degree 6 at Gauss-Lobatto points, quadrature rule of degree 13.

We have selected two test configurations: 
- "large angle" configuration: $\theta=0.9702 * \pi / 2,4$ eigenvalues computed, mesh M4L with 656 triangles (see Figure 17), leading to matrices of size $12325 \times 12325$;

- "small angle" configuration: $\theta=0.0226 * \pi / 2,12$ eigenvalues computed, mesh M64S with 6144 triangles (see Figure 18), leading to matrices of size $111265 \times 111265$.

Although the internal algorithms of the two methods are different, the parameters governing the computation of the eigenvalues are the same: a tolerance $\varepsilon$ that controls the end of the iteration process, and the dimension $N_{\text {sub }}$ of the subspaces involved in the subspace iteration $-N_{\text {sub }}$ is at least equal to $N_{\text {val }}+1$, where $N_{\text {val }}$ is the number of desired eigenvalues. We let $N_{\text {sub }}$ vary between 10 and 70 and ran the programs for $\varepsilon=10^{-n}$, $n=4,5,6,7$, while recording the number of iterations and the CPU time needed. The CPU time of the finite element part, i.e. the computation of the matrices $S$ and $M$, does not depend on these parameters. It appears on the graphs as CPUef.

The results are gathered on Figure 19 for the "large angle" configuration and on Figure 20 for the "small angle" configuration. We can observe that the number of iterations decreases as $N_{\text {sub increases. A horizontal }}$ line at the beginning of the first two graphs indicates a failed computation (no convergence after the chosen maximal number of iterations). For the values tested, the parameter $\varepsilon$ does not play a significant role on the number of iterations, nor really on the CPU time (although it does on the residual). Since the algorithms used in the two methods are not the same, the number of iterations cannot be compared directly. They are mainly a good indicator of the computation process behavior.

It is also worth to notice that the memory requirements increase as the subspace dimension $N_{\text {sub }}$ increases. This parameter is difficult to handle since it is problem dependent. These graphs suggest that there is no critical value: it can be chosen large enough to ensure computation to succeed, but not too large, mainly because of memory considerations.

The CPU graphs of the Arp method seem a bit chaotic: both algorithms need an initial vector which is chosen as a random vector by default. From our experience, we can say that this method is more sensible to this initial vector than the Mel method. Finally, these graphs show clearly that the Arp method is more efficient than the Mel method.

Remark 9.1. We are interested in the smallest eigenvalues of the problem $S w=\lambda M w$. Both methods Arp and Mel provide an option to choose in which end of the spectrum the wanted eigenvalues are to be searched. Let us mention that these algorithms perform better at computing the largest eigenvalues in general (this is due to the fact that they are ultimately based on the power method). In our case, this is typical and computation nearly always fails if the algorithms are asked to compute the smallest eigenvalues of this problem. For example, with ARPACK, we can observe the following behavior:

- in the "large angle" configuration, for $\varepsilon=10^{-4}$ and $N_{\text {sub }}=17$ or 45 , the computation fails ;

- in the "small angle" configuration, for $\varepsilon=10^{-4}$ and $N_{\text {sub }}=27$ or 43 , the computation fails ; if we change the mesh to a coarser one (M16S) with 384 triangles, the computation fails for $N_{\text {sub }}=27$ and succeeds for $N_{\text {sub }}=43$ with a CPU time of 236 sec.

Thus, the correct strategy is to compute the largest eigenvalues of the eigenvalue problem $M w=\nu S w$ and retrieve the wanted eigenvalues $\lambda$ by inversion $(\lambda=1 / \nu$ and the eigenvectors are the same). All the computations presented here have been carried on using this strategy. To conclude this remark, let us mention that the computation that took $236 \mathrm{sec}$. with the wrong strategy, takes $0.47 \mathrm{sec}$. with the right one.

\subsection{Influence of meshes and polynomial degrees in the finite element method}

We now choose the small angle $\theta=0.0226 * \pi / 2$ and fix the parameters governing the computation of the eigenvalues: $\varepsilon=10^{-6}, N_{\text {sub }}=25, N_{\text {val }}=10$, since there are 10 eigenvalues $<1$ as shown on Figure 11 .

We have built five nested meshes of the same computational domain $\Omega$, called M4S, M8S (see Figure 21), M16S, M32S (see Figure 22) and M64S (see Figure 18). The number $n$ in the name $(\mathrm{M} n \mathrm{~S}$ ) is the number of segments of the subdivision of the horizontal (and vertical) boundary of $\Omega$. It indicates the characteristic 


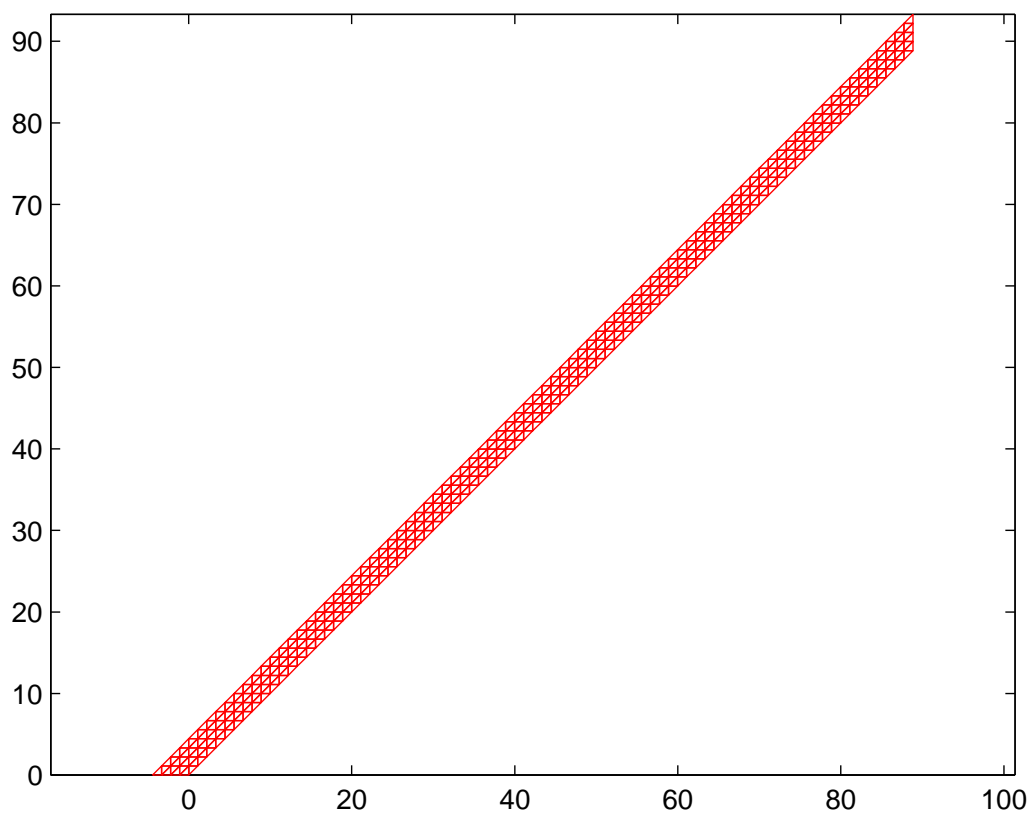

Figure 17. Mesh M4L for large angle, 656 triangles.

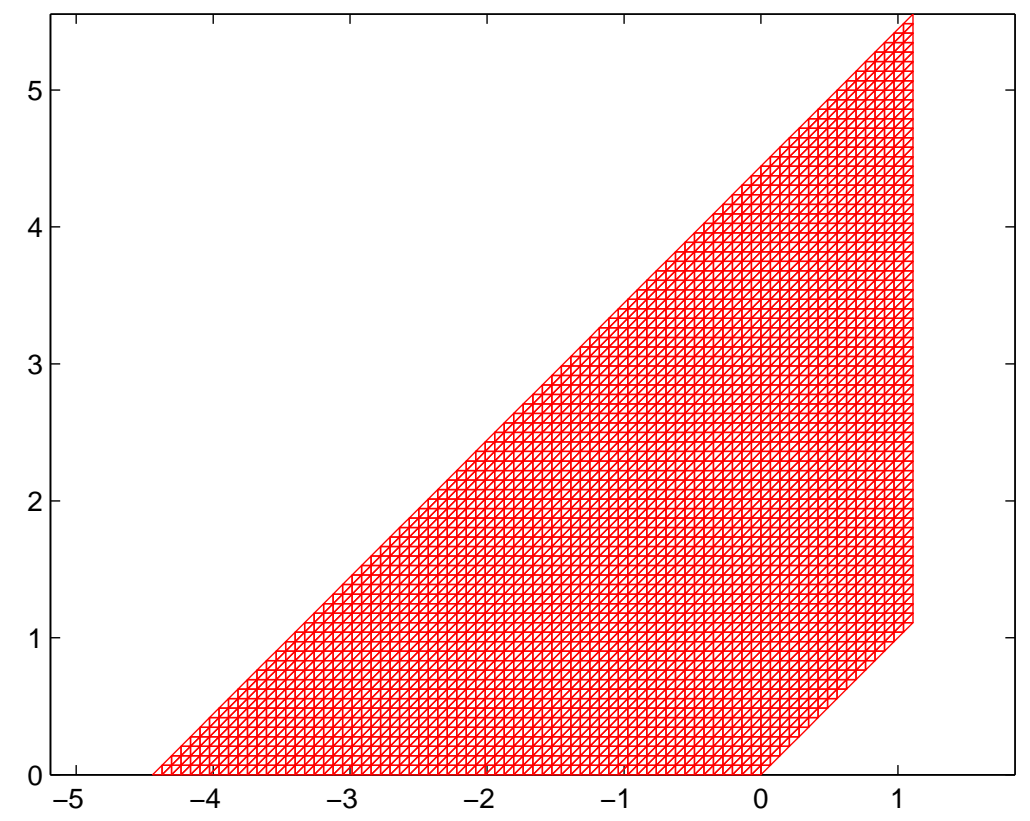

Figure 18. Mesh M64S for small angle, 6144 triangles. 

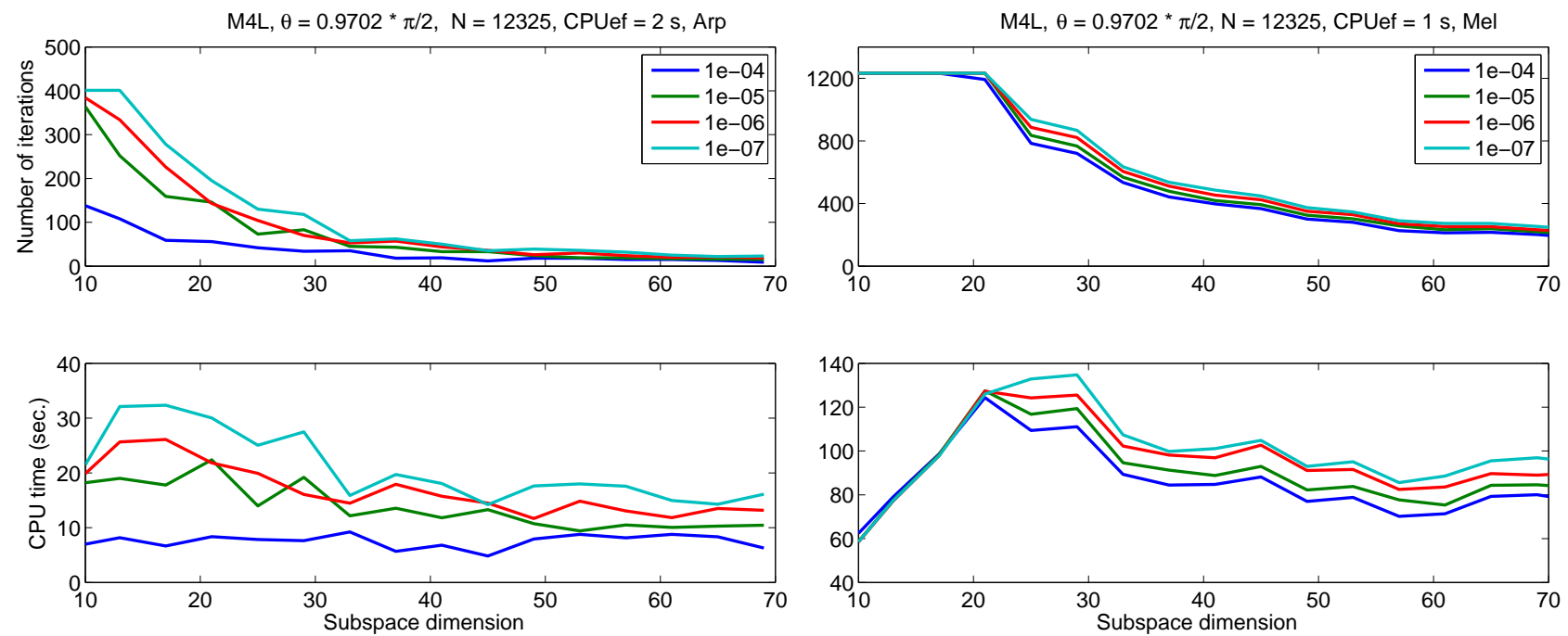

Figure 19. Comparison of the behavior of the methods for the large angle $\theta=0.9702 * \pi / 2$.
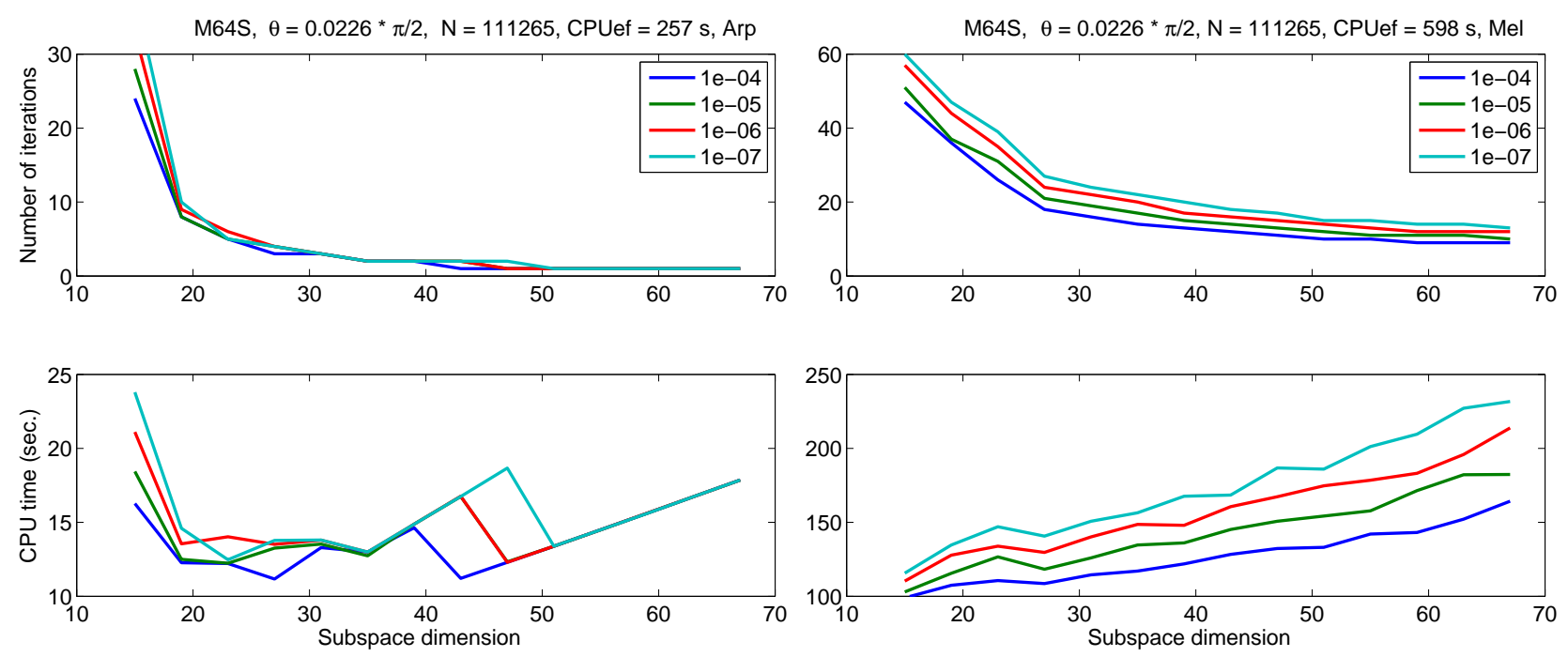

Figure 20. Comparison of the behavior of the methods for the small angle $\theta=0.0226 * \pi / 2$.

diameter $h$ of the triangles, which is halved from a mesh to the next one in the list. Thus, the number of triangles is multiplied by 4 from a mesh to the next one.

We have computed the 10 eigenvalues for $\varepsilon=10^{-8}$ using the finest mesh M64S and considered the first and last eigenvalues obtained as reference values. We denote them by $\lambda_{1}^{\text {ref }}$ and $\lambda_{10}^{\text {ref }}$.

For each mesh, we let the interpolation degree $k$ vary from 1 to 6 , while recording the differences $\left|\lambda_{1}-\lambda_{1}^{\text {ref }}\right|$ and $\left|\lambda_{10}-\lambda_{10}^{\text {ref }}\right|$ obtained for each degree. The results are gathered on Figure 23. Each point on the graphs correspond to the result of a computation. Points corresponding to the same mesh have been linked together by a line to make the graphs more readable; the corresponding degree is written below. The graphs show the convergence of the first and last computed eigenvalues: 
- with respect to the interpolation degree for a given mesh ;

- with respect to the mesh, for a given degree.

The number $N$ of degrees of freedom (d.o.f.) of the problem, which is the dimension of the matrices, is roughly proportional to $(k n)^{2}$, which explains the choice of the abscissa $\log _{10}(N) / 2$. The precision attained is about one order of magnitude better for the first eigenvalue $\lambda_{1}$ than for the last one $\lambda_{10}$. For the first eigenvalue and the coarser meshes, we observe a kind of super convergence for the small degrees, then a linear convergence. The convergence tends to be linear as the mesh becomes finer. For the last eigenvalue $\lambda_{10}$, the convergence is mainly linear.
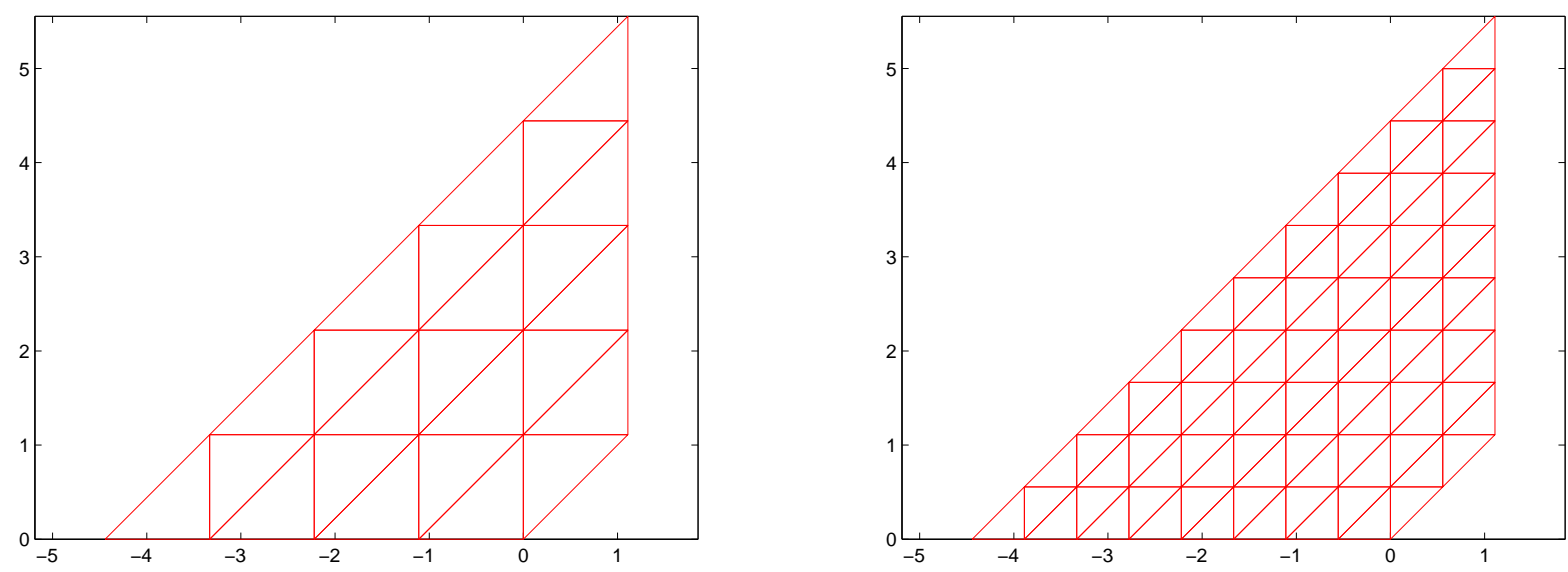

Figure 21. Meshes M4S (24 triangles) and M8S (96 triangles).
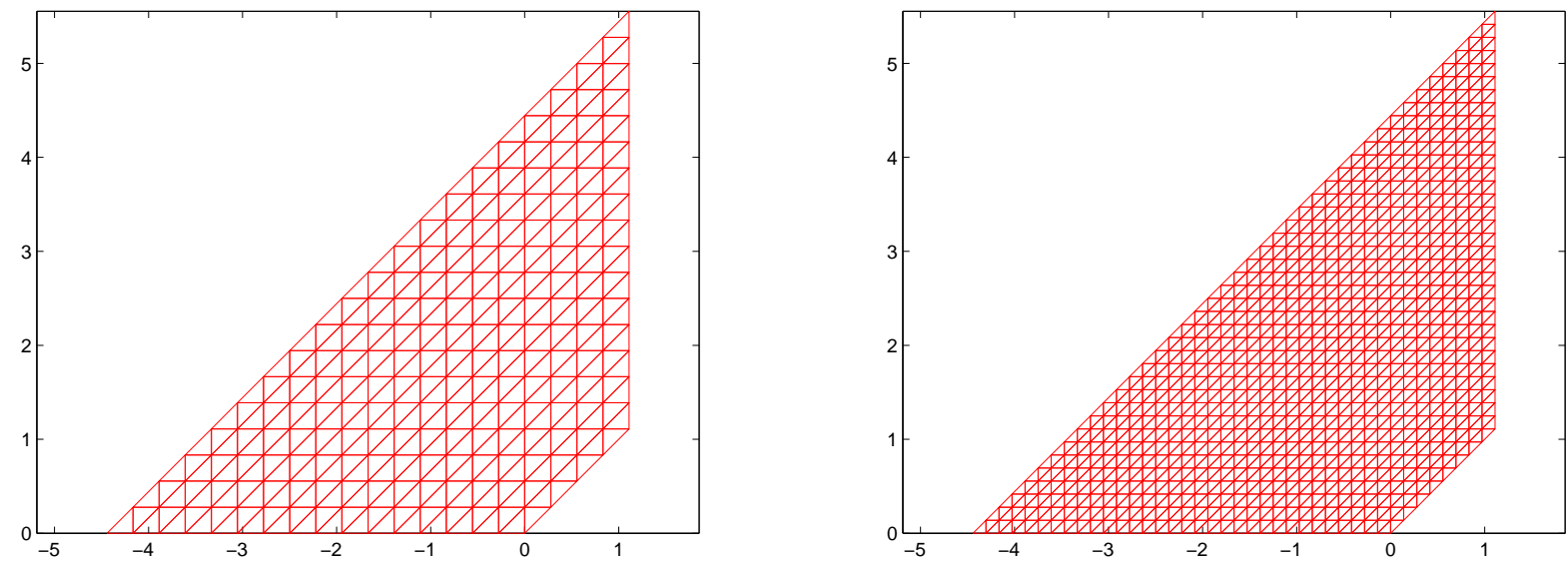

Figure 22. Meshes M16S (384 triangles) and M32S (1536 triangles).

This phenomenon of super convergence is due to a sort of locking which takes place in coarser meshes: such meshes with low degree $k$ are not able to capture the fine scale structure of the eigenvectors (see in Figure 13 representations of the first eigenvector in the computational domain for small angles). 

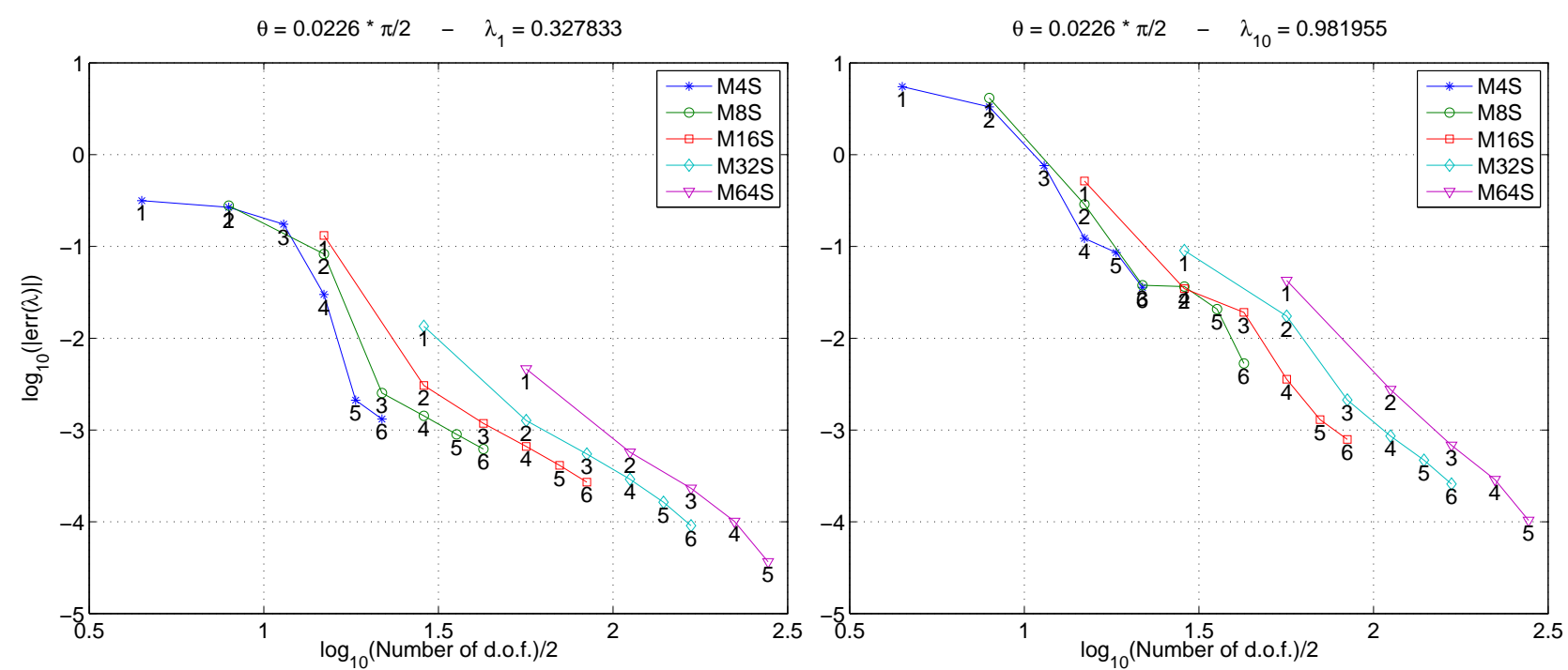

FiguRE 23. Convergence towards $\lambda_{1} \simeq 0.32783$ and $\lambda_{10} \simeq 0.98195$.

The average rate of convergence with respect to $k^{-1}$ is twice the rate of convergence with respect to $h$, in accordance with a well-known convergence result in finite elements [31]. Typically the rate is 1 in $h$ and 2 in $k^{-1}$. These somewhat low rates are due to the singularity at the reentrant corner of $\Omega$. This singularity comes from the Laplace singularity (9), and for $\theta=0.0226 * \pi / 2$, the singularity exponent $\frac{\pi}{\omega}$ is $\simeq 0.5057$.

Nevertheless, all our computations are accurate enough to display clearly the asymptotic behavior of the eigenpairs in the small angle and large angle limits, see Figure 16. 


\section{REFERENCES}

[1] S. Agmon. Lectures on exponential decay of solutions of second-order elliptic equations: bounds on eigenfunctions of $N$-body Schrödinger operators, volume 29 of Mathematical Notes. Princeton University Press, Princeton, NJ 1982.

[2] Y. Avishai, D. Bessis, B. G. Giraud, G. Mantica. Quantum bound states in open geometries. Phys. Rev. B 44(15) (Oct 1991) 8028-8034.

[3] V. Bonnaillie, M. Dauge, N. Popoff, N. Raymond. Discrete spectrum of a model schrödinger operator on the half-plane with neumann conditions. To appear in ZAMP (2011).

[4] D. Borisov, P. Freitas. Asymptotics of Dirichlet eigenvalues and eigenfunctions of the Laplacian on thin domains in $\mathbb{R}^{d} . J$. Funct. Anal. 258 (2010) 893-912.

[5] J. P. Carini, J. T. Londergan, K. Mullen, D. P. Murdock. Multiple bound states in sharply bent waveguides. Phys. Rev. B 48(7) (Aug 1993) 4503-4515.

[6] B. Chenaud, P. Duclos, P. Freitas, D. KrejČIrí́k. Geometrically induced discrete spectrum in curved tubes. Differential Geom. Appl. 23(2) (2005) 95-105.

[7] H. L. Cycon, R. G. Froese, W. Kirsch, B. Simon. Schrödinger operators with application to quantum mechanics and global geometry. Texts and Monographs in Physics. Springer-Verlag, Berlin, study edition 1987.

[8] M. Dauge, B. Helffer. Eigenvalues variation. I. Neumann problem for Sturm-Liouville operators. J. Differential Equations 104(2) (1993) 243-262.

[9] M. Dauge, N. Raymond. Plane waveguides with corners: small angle limit. Preprint (2011).

[10] P. Duclos, P. Exner. Curvature-induced bound states in quantum waveguides in two and three dimensions. Rev. Math. Phys. 7(1) (1995) 73-102.

[11] P. Exner, P. ŠEba, P. ŠŤovíčeK. On existence of a bound state in an L-shaped waveguide . Czech. J. Phys. 39(11) (1989) $1181-1191$.

[12] P. Exner, M. Tater. Spectrum of Dirichlet Laplacian in a conical layer. J. Phys. A43 (2010).

[13] S. Fournais, B. Helffer. Spectral methods in surface superconductivity. Progress in Nonlinear Differential Equations and their Applications, 77. Birkhäuser Boston Inc., Boston, MA 2010.

[14] P. Freitas. Precise bounds and asymptotics for the first Dirichlet eigenvalue of triangles and rhombi . J. Funct. Anal. 251 (2007) 376-398.

[15] L. Friedlander, M. Solomyak. On the spectrum of narrow periodic waveguides. Russ. J. Math. Phys. 15(2) (2008) $238-242$.

[16] L. Friedlander, M. Solomyak. On the spectrum of the Dirichlet Laplacian in a narrow strip. Israel J. Math. 170 (2009) $337-354$.

[17] F. M. Gomes, D. C. Sorensen. Arpack++, object-oriented package designed to solve large scale eigenvalue problems. http://www.ime.unicamp.br/ chico/arpack++/(2000).

[18] P. Grisvard. Boundary Value Problems in Non-Smooth Domains. Pitman, London 1985.

[19] B. Helffer. Semi-classical analysis for the Schrödinger operator and applications, volume 1336 of Lecture Notes in Mathematics. Springer-Verlag, Berlin 1988.

[20] B. Helffer, J. Sjöstrand. Multiple wells in the semiclassical limit. I. Comm. Partial Differential Equations 9(4) (1984) $337-408$.

[21] T. Kato. Perturbation theory for linear operators. Classics in Mathematics. Springer-Verlag, Berlin 1995. Reprint of the 1980 edition.

[22] V. A. Kondrat'Ev. Boundary-value problems for elliptic equations in domains with conical or angular points. Trans. Moscow Math. Soc. 16 (1967) 227-313.

[23] P. LÉvy-Bruhl. Introduction à la théorie spectrale. Sciences Sup. Dunod, Paris 2003.

[24] J.-L. Lions, E. Magenes. Problèmes aux limites non homogènes et applications. Vol. 1. Travaux et Recherches Mathématiques, No. 17. Dunod, Paris 1968.

[25] D. Martin. Mélina, bibliothèque de calculs éléments finis. http://anum-maths.univ-rennes1.fr/melina (2010).

[26] A. Martinez. Développements asymptotiques et effet tunnel dans l'approximation de Born-Oppenheimer. Ann. Inst. H. Poincaré Phys. Théor. 50(3) (1989) 239-257.

[27] A. Morame, F. Truc. Remarks on the spectrum of the Neumann problem with magnetic field in the half-space. J. Math. Phys. 46(1) (2005) 012105, 13.

[28] T. Ourmières. Guides d'ondes quantiques (thèse de doctorat). En cours. (2011-).

[29] A. Persson. Bounds for the discrete part of the spectrum of a semi-bounded Schrödinger operator. Math. Scand. 8 (1960) $143-153$.

[30] M. Reed, B. Simon. Methods of modern mathematical physics. I. Academic Press Inc. [Harcourt Brace Jovanovich Publishers], New York, second edition 1980. Functional analysis.

[31] C. Schwab. $p$ - and hp-finite element methods. Theory and applications in solid and fluid mechanics. The Clarendon Press Oxford University Press, New York 1998. 